\title{
Use of Thin Sectioning (Nanoskiving) to Fabricate Nanostructures for Electronic and Optical Applications
}

\section{Citation}

Lipomi, Darren J., Ramses V. Martinez, and George M. Whitesides. 2011. "Use of Thin Sectioning (Nanoskiving) to Fabricate Nanostructures for Electronic and Optical Applications." Angewandte Chemie International Edition 50, no. 37: 8566-8583.

\section{Published Version}

doi:10.1002/anie.201101024

\section{Permanent link}

http://nrs.harvard.edu/urn-3:HUL.InstRepos:12967815

\section{Terms of Use}

This article was downloaded from Harvard University's DASH repository, and is made available under the terms and conditions applicable to Open Access Policy Articles, as set forth at http:// nrs.harvard.edu/urn-3:HUL.InstRepos:dash.current.terms-of-use\#OAP

\section{Share Your Story}

The Harvard community has made this article openly available.

Please share how this access benefits you. Submit a story.

\section{Accessibility}


Use of Thin Sectioning (Nanoskiving) to Fabricate Nanostructures for Electronic and

\section{Optical Applications}

Darren J. Lipomi, ${ }^{1}$ Ramses V. Martinez, ${ }^{1}$ and George M. Whitesides ${ }^{1,2}$

${ }^{1}$ Department of Chemistry and Chemical Biology, Harvard University, 12 Oxford St.,

Cambridge, MA 02138, USA

${ }^{2}$ Kavli Institute for Bionanoscience and Technology, School of Engineering and Applied

Sciences, Harvard University, 29 Oxford St., Cambride, MA 02138, USA

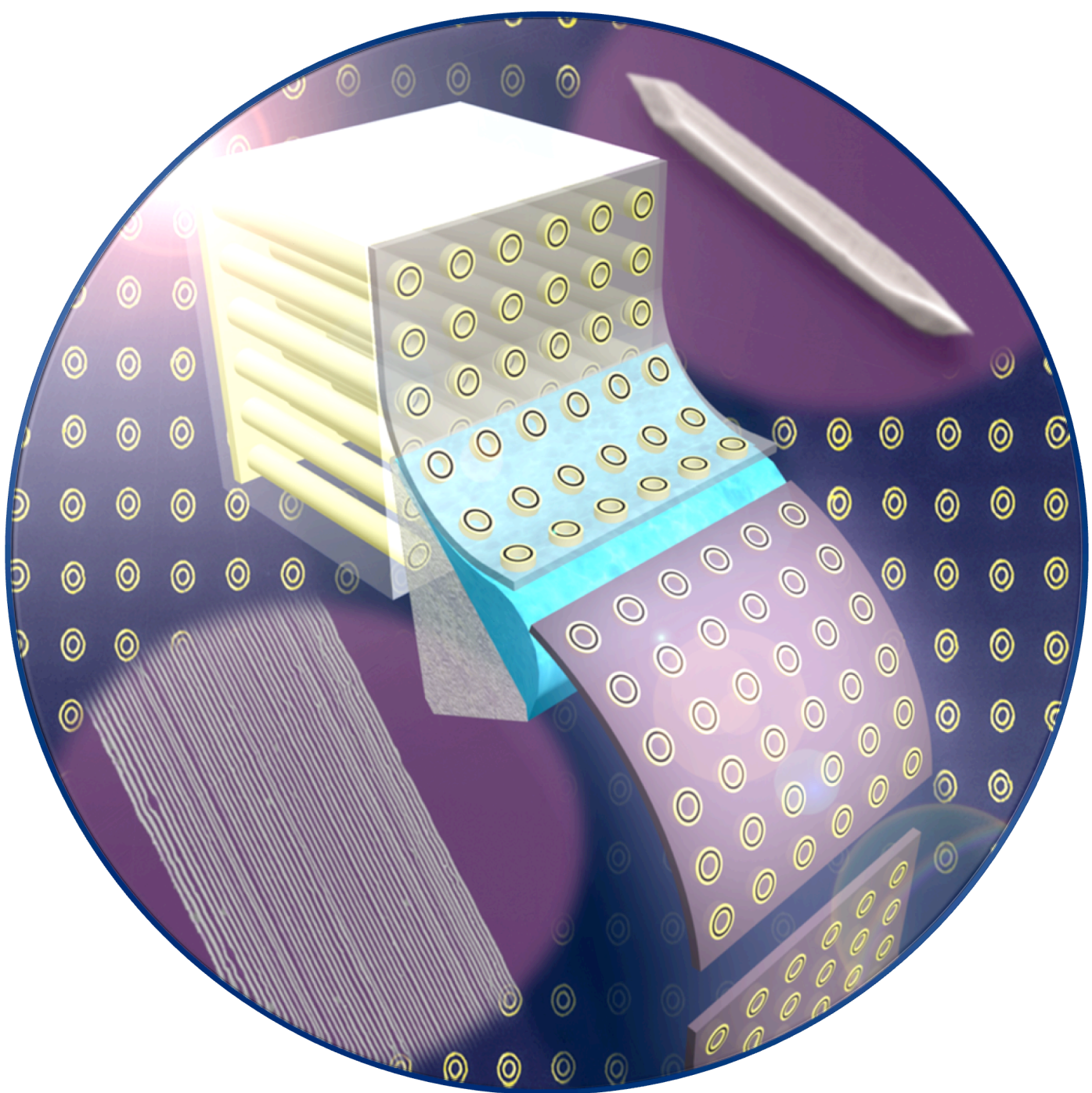

Keywords: nanoskiving, nanofabrication, soft lithography, plasmonics, ultramicrotomy 


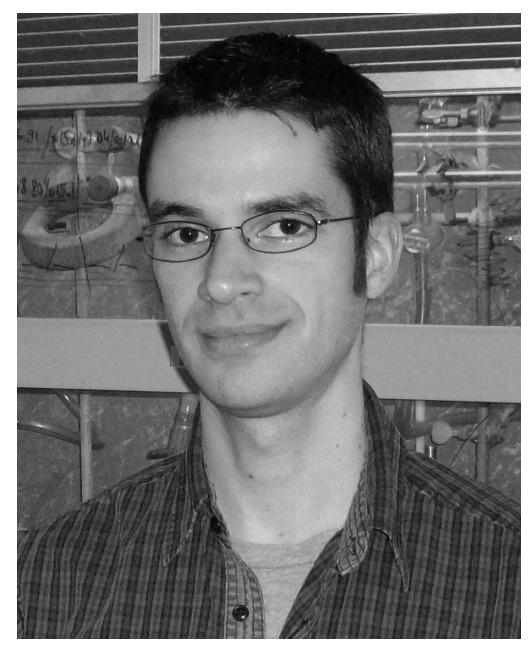

Darren J. Lipomi was born in Rochester, New York, in 1983. He earned his B.A. in chemistry, with a minor in physics, from Boston University in 2005. Under Prof. James S. Panek, his research focused on the total synthesis of natural products and asymmetric reaction methodology. He earned his A.M. and Ph.D. in chemistry at Harvard University in 2008 and 2010, with Prof. George M. Whitesides. At Harvard, he developed several unconventional approaches to fabricate micro- and nanostructures for electronic and optical applications. He is now an Intelligence Community Postdoctoral Fellow in the Department of Chemical Engineering at Stanford University.

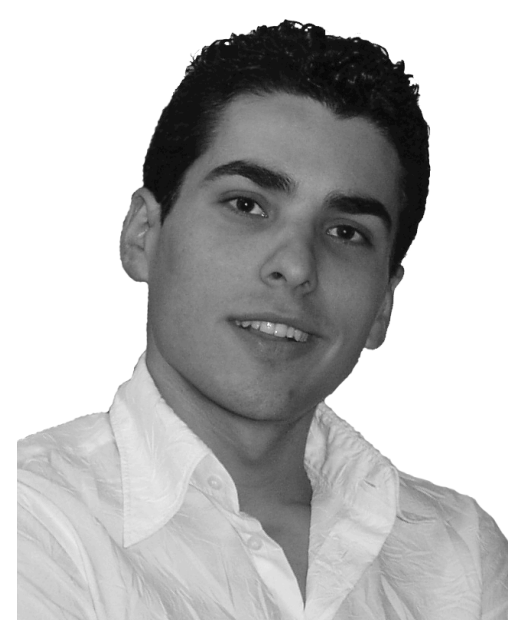

Ramsés V. Martínez was born in Madrid in 1981. He completed his Bachelor's and Master's degrees in physics at Universidad Autónoma de Madrid in June 2004. In 2009, He received his PhD degree from the Spanish High Council of Scientific Research (CSIC) under the supervision of Prof. R. García. He is currently a postdoctoral researcher in Prof. George M. Whitesides' group at Harvard University. His current research focuses on the development of new simple and low-cost methods of nanofabrication. 


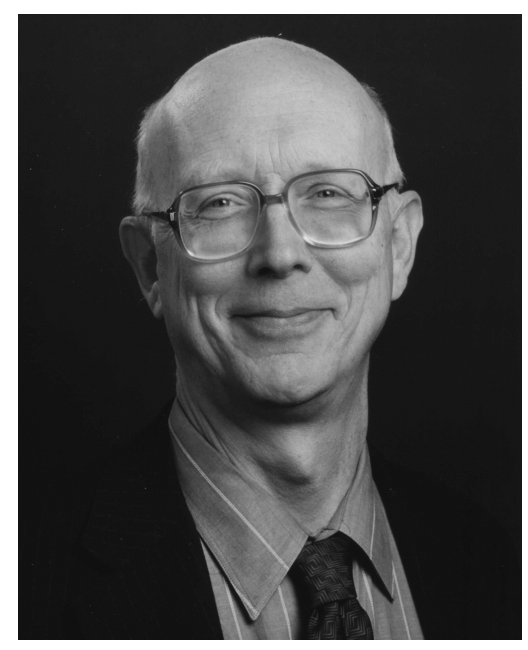

George M. Whitesides received his A.B. degree from Harvard University in 1960 and his Ph.D. degree from the California Institute of Technology in 1964. A Mallinckrodt Professor of Chemistry from 1982 to 2004, he is now a Woodford L. and Ann A. Flowers University Professor. Prior to joining the Harvard faculty, he was a member of the chemistry faculty of the Massachusetts Institute of Technology. His research interests include physical and organic chemistry, materials science, biophysics, complexity, surface science, microfluidics, selfassembly, micro- and nanotechnology, and cell-surface biochemistry.

\section{Lead-In}

This paper reviews nanoskiving - a simple and inexpensive method of nanofabrication, which minimizes requirements for access to cleanrooms and associated facilities, and which make it possible to fabricate nanostructures from materials, and of geometries, to which more familiar methods of nanofabrication are not applicable. Nanoskiving requires three steps: i) deposition of a metallic, semiconducting, ceramic, or polymeric thin film onto an epoxy substrate; ii) embedding this film in epoxy, to form an epoxy block, with the film as an inclusion; and iii) sectioning the epoxy block into slabs with an ultramicrotome. These slabs, which can be $30 \mathrm{~nm}-10 \mu \mathrm{m}$ thick, contain nanostructures whose lateral dimensions are equal to the thicknesses of the embedded thin films. Electronic applications of structures produced by this method include nanoelectrodes for electrochemistry, chemoresistive nanowires, and 
heterostructures of organic semiconductors. Optical applications include surface plasmon resonators, plasmonic waveguides, and frequency-selective surfaces.

\section{Introduction}

\subsection{Why Nano?}

Many of the most important phenomena in nature-e.g., the binding of proteins and ligands, the absorption of light by molecules, and the mean free path of electrons in metalsinvolve forces or processes operating over distances of $1-100 \mathrm{~nm}$. Processes that occur over this range - which begins with large molecules, and ends with objects that are resolved with conventional microscopes - are the purview of the field known as "nanoscience". Nanoscience represents an extension of, and the overlap between, the chemistry of materials, solid-state physics, electrical and mechanical engineering, biology, and other fields. Nanostructured materials display properties not found in bulk materials. These properties include the effects of size confinement, including size-dependent band gaps in quantum dots, ${ }^{[1]}$ localized surface plasmon resonances in metallic nanoparticles, ${ }^{[2]}$ exceptional strength and ballistic transport of electrons in carbon nanotubes, ${ }^{[3]}$ and consequences of the fact that these structures are "all—or mostly-surface". ${ }^{[4]}$

Beyond discovery-based scientific inquiry in these areas, there are also opportunities for technological development. Nanostructured materials have already enabled the electronics industry to fabricate faster, cheaper, and more efficient devices, ${ }^{[5]}$ are making inroads into medicine, ${ }^{[6]}$ and could contribute in significant ways to sensing, communication, and computation based on nanophotonics. ${ }^{[7,8]}$ Developing methods of generating and patterning nanostructures that are reproducible, scalable, inexpensive, applicable to different types of 
materials, and as widely accessible to as many users as possible, is thus an important motivation for the sciences of materials chemistry and nanofabrication.

\subsection{Nanofabrication}

Nanofabrication refers to the generation of patterns whose individual elements have at least one lateral dimension between approximately $1 \mathrm{~nm}$ and $100 \mathrm{~nm} .{ }^{[9]}$ Nanofabrication, along with microfabrication before it, has been a key enabler of modern science and technology, and has underpinned essentially all electronics since the invention of the integrated circuit in $1958 .^{[5]}$ Nanofabrication, as practiced in electronics and related areas of technology, has two principal steps: mastering (e.g., forming master structures such as amplitude and phase masks for photolithography) and replication. ${ }^{[9]}$ Mastering encodes nanoscale information about patterns in a form from which the pattern can be replicated (usually with a reduction in lateral dimensions). In semiconductor manufacturing, the principal tool for mastering is electron-beam lithography (EBL), which creates patterns in a photomask. Mastering is a time-intensive and expensive process, and may require twenty hours to produce a single mask ${ }^{[10]}$ Replication of this pattern takes the form of photolithography, in which light passes through the photomask and creates an image on a wafer coated with a film of a light-sensitive polymer called a photoresist. Modern exposure tools generate around 100 copies $\min ^{-1} .{ }^{[10]}$ After chemical processing, the surface of the material comprising the wafer can be modified in the areas of the film unprotected by photoresist (e.g., by etching, vapor deposition, ion implantation, or other processes). Iteration of these processes generates the devices and connections on a chip.

An empirical trend-Moore's Law—shows that the number of transistors per microprocessor has doubled approximately every 18 months, with concomitant decreases in cost 
and power consumption, and increases in speed for information processors and in storage capacity for memory devices. ${ }^{[11,12]}$ This trend has become a self-fulfilling prophecy, which has motivated the development of new steppers for projection photolithography, ${ }^{[13]}$ chemistry for photoresists, ${ }^{[14]}$ and other technologies. ${ }^{[5]}$ The state-of-the-art in photolithography produces features with an average half-pitch in memory devices of $32 \mathrm{~nm}$ using $193 \mathrm{~nm}$ light combined with immersion optics, ${ }^{[15]}$ phase-shifting masks, ${ }^{[16]}$ and multiple exposures. ${ }^{[17]}$ Next-generation lithographic tools, including extreme ultraviolet lithography (EUVL), ${ }^{[18]}$ maskless lithography (ML2, which would use thousands of electron beams to replicate patterns without the need for a physical master), ${ }^{[19]}$ and step-and-flash imprint lithography (SFIL) ${ }^{[20]}$ are expected to drive the average half-pitch down to $16 \mathrm{~nm}$ by 2019 , according to the International Technical Roadmap for Semiconductors. ${ }^{[10]}$ Informed speculation suggests an ultimate limit that may be as small as 8 $\mathrm{nm}$.

Semiconductor devices are manufactured using the most sophisticated processes ever employed for commercial products. The scale of investment in these tools is so high (and the precision in replication is so precise), that it does not make sense to compete with them for their designed purpose - manufacturing multilayered semiconductor devices on planar, rigid substrates. There are, however, at least five reasons to explore "unconventional" methods of fabrication. (1) Cost: photolithographic steppers and other tools can be prohibitively expensive, particularly for universities. ${ }^{[21]}$ (2) Accessibility: scanning-beam lithographic and photolithographic tools are usually found in a cleanroom, whose construction, operation, and maintenance impose a significant financial burden on an institution. (3) Incompatibility: organics, biologics, and other materials not normally used in (or compatible with) electronic devices often cannot be patterned directly using conventional tools, nor can they by processed 
using the same equipment, or in the same cleanroom, as that used for electronics. (4) Form factors: conventional tools are incompatible with non-planar, ${ }^{[2]}$ mechanically compliant, ${ }^{[23]}$ or very small $(<100 \mu \mathrm{m})$ substrates. ${ }^{[24]}(5)$ Overkill: there are a large number of potential applications of nanotechnology — in biology, optics, chemistry, devices for the conversion and storage of energy, and other areas - that are significantly more tolerant of defects than are semiconductor devices, and whose requirements can be satisfied using simpler tools.

\subsection{Soft Lithography}

Soft lithography ${ }^{[25]}$ is a set of techniques whose key step is the transfer of patterns by printing or molding, usually using an elastomeric stamp or mold, which often is made from poly(dimethylsiloxane) (PDMS), perfluoropolyethers, or other polymers. ${ }^{[26]}$ There are three general modes of soft lithography: i) molding (replica molding, ${ }^{[2,27,28]}$ solvent-assisted micromolding, ${ }^{[29]}$ and micromolding in capillaries ${ }^{[30]}$ ); ii) printing (microcontact printing, ${ }^{[31-34]}$ charge printing, ${ }^{[35]}$ and nanotransfer printing ${ }^{[36]}$ ); and iii) near-field optical lithography (in two or three dimensions). ${ }^{[37-39]}$ The key steps of all forms of soft lithography rely on physical contact. The limits of fidelity in replication in soft lithography are not determined by the diffraction of light, or scattering of beams of charged particles, but rather by van der Waals interactions, physical deformation of the stamp, wicking or spreading of liquid inks, and related processes. ${ }^{[9]}$ Nanoimprint lithography (NIL) and step-and-flash imprint lithography (SFIL) — techniques pioneered by $\mathrm{Chou}^{[9,40,41]}$ and Willson ${ }^{[20,42]}$ _ largely circumvent errors due to mechanical deformation by using hard masters.

Unconventional approaches to nanofabrication that do not involve a stamp or mold include variants of lithography using scanning probe tools. ${ }^{[43]}$ Dip-pen nanolithography, invented 
by Mirkin and coworkers, uses an atomic force microscope (AFM) tip dipped in "ink" (e.g., small molecules, polymers, or other materials) to draw patterns on surfaces with linewidths as small as $10 \mathrm{~nm}$ with $\sim 5 \mathrm{~nm}$ spatial resolution. ${ }^{[44]}$ This method operates using single AFM tips or arrays of thousands of tips connected in parallel. ${ }^{[45]}$ Related techniques include local oxidation, nanoshaving and nanografting using an AFM tip. ${ }^{[46]}$ Indentation lithography using the diamond tip of a commercial nanoindentation system can produce patterns in hard materials, such as silicon dioxide. ${ }^{[47]}$

One of the most effective strategies for developing new approaches to nanofabrication is the adaptive re-use of analytical tools for the purposes of fabrication. In the same way that photolithography and EBL have their bases in optical and electron-beam microscopy, dip-pen nanolithography has its basis in AFM, and indentation lithography has its basis in nanoindentation of thin films and coatings. ${ }^{[47]}$ The ultramicrotome is a tool capable of sectioning materials into slabs as thin as $10 \mathrm{~nm},{ }^{[48]}$ but the use of this fact has been, until recently, restricted to its original purpose: sectioning samples (usually biological or polymeric) for examination using an electron microscope ${ }^{[49]}$ In a new method of nanofabrication that we have named "nanoskiving," ultramicrotomy can be used alone, or in combination with photolithography or soft lithography, to produce patterns of nanostructures of materials and in geometries that are difficult or impossible to produce using other means. The tools required (the only necessary one is the ultramicrotome) and the means to prepare thin films, and to carry out low-resolution photolithography and soft lithographic molding, are generally less expensive and more accessible than conventional tools for fabricating nanostructures. ${ }^{[50]}$

\section{Ultramicrotomy and Nanoskiving}




\subsection{Microtomy and Microscopy}

Sectioning with a microtome has been a tool of microscopists since John Hill described the first instrument in $1770 .{ }^{[51]}$ This manually operated device could produce sections of timber as thin as $25 \mu \mathrm{m}$, for analysis with a light microscope ${ }^{[51]}$ Use of the microtome was restricted, for the most part, to biology, until the invention of the transmission electron microscope (TEM) in the 1930s. ${ }^{[52]}$ Transmission of electrons through a specimen required a device capable of producing sections with thicknesses $<100 \mathrm{~nm}$. This device became known as the ultramicrotome. Ultramicrotomy enabled microstructural analysis not only of biological specimens, but of inorganic materials as well. It is the primary method for the preparation of polymeric samples for TEM, and is complementary to ion thinning and electropolishing for the preparation of hard materials. ${ }^{[48]}$

Several books and reviews describe the history of microtomy. Bracegirdle's book describes the development of microtomy between 1770 and $1910{ }^{[53]}$ The review by Pease and

Porter provides an account of the co-development of electron microscopy and ultramicrotomy, ${ }^{[52]}$ while that of Malis and Steele is the most complete review of ultramicrotomy, in the context of inorganic materials science, through $1990 .{ }^{[48]}$ The book by Goldstein et al. covers all aspects of embedding and sectioning hard and soft materials, including histological samples. ${ }^{[54]}$

\subsection{The Ultramicrotome}

Figure 1a shows a modern ultramicrotome. Its components include a stereomicroscope, a movable stage that holds the knife, and a sample chuck (1b) attached to a movable arm that holds the epoxy block. The movable arm controls the fine positioning of the block and can advance in steps as small as $1 \mathrm{~nm}$ toward a single-crystalline diamond knife (1c). The mechanism of fine 
control involves a stepper motor connected to a spindle, and a lever that transforms micrometerlength displacements of the spindle into nanometer-length displacements of the arm. ${ }^{[50]}$ The arm and the epoxy block advance toward the knife in an elliptical path when viewed from the side, as drawn. The speed of cutting is $0.1-10 \mathrm{~mm} / \mathrm{s}$, and produces sections at a rate of $0.5-2 \mathrm{~s}^{-1}$.
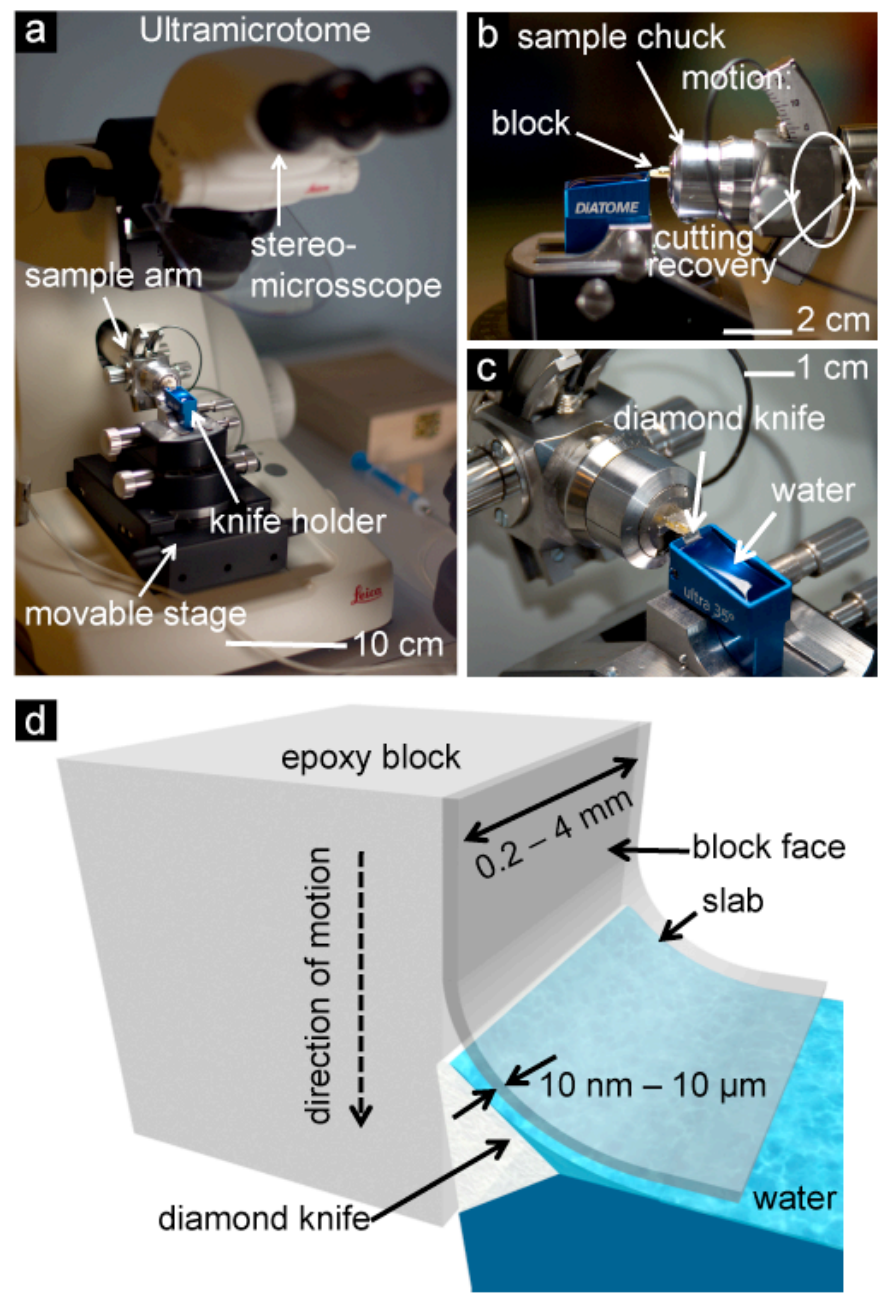

Figure 1. Photographs and schematic drawings of the tools of ultramicrotomy and nanoskiving. (a) A photograph of a Leica UC6 ultramicrotome. (b) A side view of the sample chuck and knife holder as the epoxy block impinges upon the knife. (c) A top view of the single-crystalline diamond blade and the water-filled trough. Photographs courtesy of Dr. Ryan Chiechi. (d) A schematic drawing of the sectioning process. The epoxy block contacts the diamond knife, and the offcut (the "slab") slides onto the surface of water. The cutting process repeats until the user stops the ultramicrotome or the embedded material is consumed. The water supports the slabs until the user collects them.

\subsection{The Process of Sectioning}


Figure 1d is a schematic drawing of the process of sectioning, which involves a complicated interplay of events: compression of the sample during the initiation of cutting, and of the slab thereafter; tension perpendicular to the plane of sectioning; generation of new surfaces; bending, as the slab reorients from vertical to horizontal; shearing stress (greatest in materials with low flexibility); friction of the slabs on the knife; and generation of heat. ${ }^{[55]}$ The nascent epoxy slabs slide onto the surface of a water bath in the form of individual slabs or ribbons of connected slabs. ${ }^{[56]}$

There are two general mechanisms of the process of sectioning proposed in the literature of ultramicrotomy: i) true sectioning, in which the edge of the knife maintains contact with both the bottom surface of the nascent slab and the facet of the block, and ii) crack initiation and propagation. ${ }^{[57]}$ True sectioning appears to dominate for metals and alloys. Characteristics of true sectioning include the appearance of shear lamellae and perpendicular to the direction of cutting and concomitant compression along the same axis. Microtomed specimens-as well as micromachined chips of metal—exhibit these same characteristics. ${ }^{[48]}$ Mechanisms resembling crack initiation and propagation appear to operate in brittle materials, such as minerals and ceramics. ${ }^{[48]}$ The orientation of cleavage planes in crystalline samples also determines the extent of fragmentation upon sectioning brittle materials, as Antonovsky observed in samples of alumina ${ }^{[58]}$ Malis found that true sectioning and crack initiation and propagation can operate simultaneously on different grains in the same sample, as he observed in sections of highstrength steel. ${ }^{[48]}$

\subsection{The Embedding Medium}


Most forms of ultramicrotomy—and all forms of nanoskiving — require an embedding medium to support the embedded structures during sectioning. In nanoskiving, the embedding medium also preserves the orientations of the nanostructures within the slabs. The embedding medium should have two properties, i) a relatively high value of elastic modulus ( $\sim 3 \mathrm{GPa}$; materials that are too compliant deflect from the knife edge, rather than cleave), and ii) a high yield stress, only after which the material undergoes plastic deformation ( $70 \mathrm{MPa}$; otherwise the slab will deform upon sectioning). ${ }^{[55]}$ Crosslinked epoxy resins fill most of these criteria at ambient temperatures, although it is possible to section softer materials at cryogenic temperatures. Our laboratory has achieved excellent results with UVO-114 (Epotek), which is UV-curable, ${ }^{[59]}$ and good results with Araldite $502^{[60]}$ and Epo-Fix (Electron Microscopy

Sciences),${ }^{[61]}$ which are thermally curable. The newly cut surfaces of the epoxy slabs are smooth, with values of roughness $(\mathrm{rms})$ of $\sim 0.5 \mathrm{~nm}^{[62]}$

\subsection{Diamond Knives}

The knife is the most important component of the ultramicrotome. While glass knives are inexpensive and disposable, the cutting edge degrades rapidly (after a few uses). Our laboratory uses a $35^{\circ}$ diamond knife, $1.8-2.4 \mathrm{~mm}$ in length, whose edge has a radius of curvature of $3-6$ $\mathrm{nm} \cdot{ }^{[63]}$ The cost of a knife is $\$ 2,000-\$ 3,000$. Knives must be re-sharpened every $6-12$ months; this service is about half the cost of a new knife. In our experience, damage to the knife takes the form of chipping (rather than homogeneous "dulling"). Chips in the knife cause scoring of the epoxy slabs in the direction of cutting. Most scores are ten to a few hundred nm wide. The most rapid deterioration of the quality of a knife we have observed occurred when sectioning thick films ( $\sim 500 \mathrm{~nm})$ of hard materials (e.g. Ti) and micron-scale ceramic objects (e.g. optical fibers). 
Significant chipping of the knife also occurs when hard inorganic dust particles become inadvertently embedded in the epoxy blocks.

\subsection{Nanoskiving Thin Films into Nanowires}

The simplest embedded structure that can be sectioned using nanoskiving is a metallic, ceramic, semiconducting, polymeric, or other planar thin film (Figure 2). Sectioning a thin film produces a nanowire with a (roughly) rectangular cross section whose width is determined by the thickness of the film, and whose height is determined by the ultramicrotome. To determine the applicability of different materials to nanoskiving, we performed a survey of thin films, deposited using different methods: evaporation, sputter-coating, chemical-vapor deposition, electroless deposition, deposition in an electrochemical cell, spin-coating, and solution-phase synthesis and subsequent deposition. The four major conclusions are: i) for evaporated, elemental films, soft and compliant materials (softer than platinum, or those with bulk values of hardness $<500 \mathrm{MPa}$ ) tend to remain intact upon sectioning, while hard and stiff materials (harder than nickel) tend to fragment; ii) platinum and nickel are on the borderline between soft and hard, for which the extent of fragmentation depends on the method of deposition, and the morphology of the film; iii) the extent of fragmentation is higher when the orientation of the film is parallel to the direction of cutting than when the film is perpendicular to it; and iv) the speed of cutting has no effect on the frequency of defects, from $0.1 \mathrm{~mm} / \mathrm{s}$ to $10 \mathrm{~mm} / \mathrm{s}$ (which is consistent with Jesior's observation that the cutting speed also has no effect on compression. ${ }^{[64,57]}$ We have successfully formed nanostructures of aluminum, copper, silver, gold, lead, bismuth, palladium, platinum, nickel, germanium, silicon dioxide, all conducting and semiconducting polymers tested, and films of lead sulfide nanocrystals. Figure 3a-3d show examples of four 
classes of thin films successfully sectioned into nanowires that were intact over $100 \mu \mathrm{m}$ : metallic (gold, 3a), ceramic (silicon dioxide, 3b), semiconducting (germanium, 3c), and polymeric (poly(3-hexylthiophene) (P3HT), 3d).

1) Deposit metallic, polymeric, semiconducting or oxide film on flat epoxy substrate:

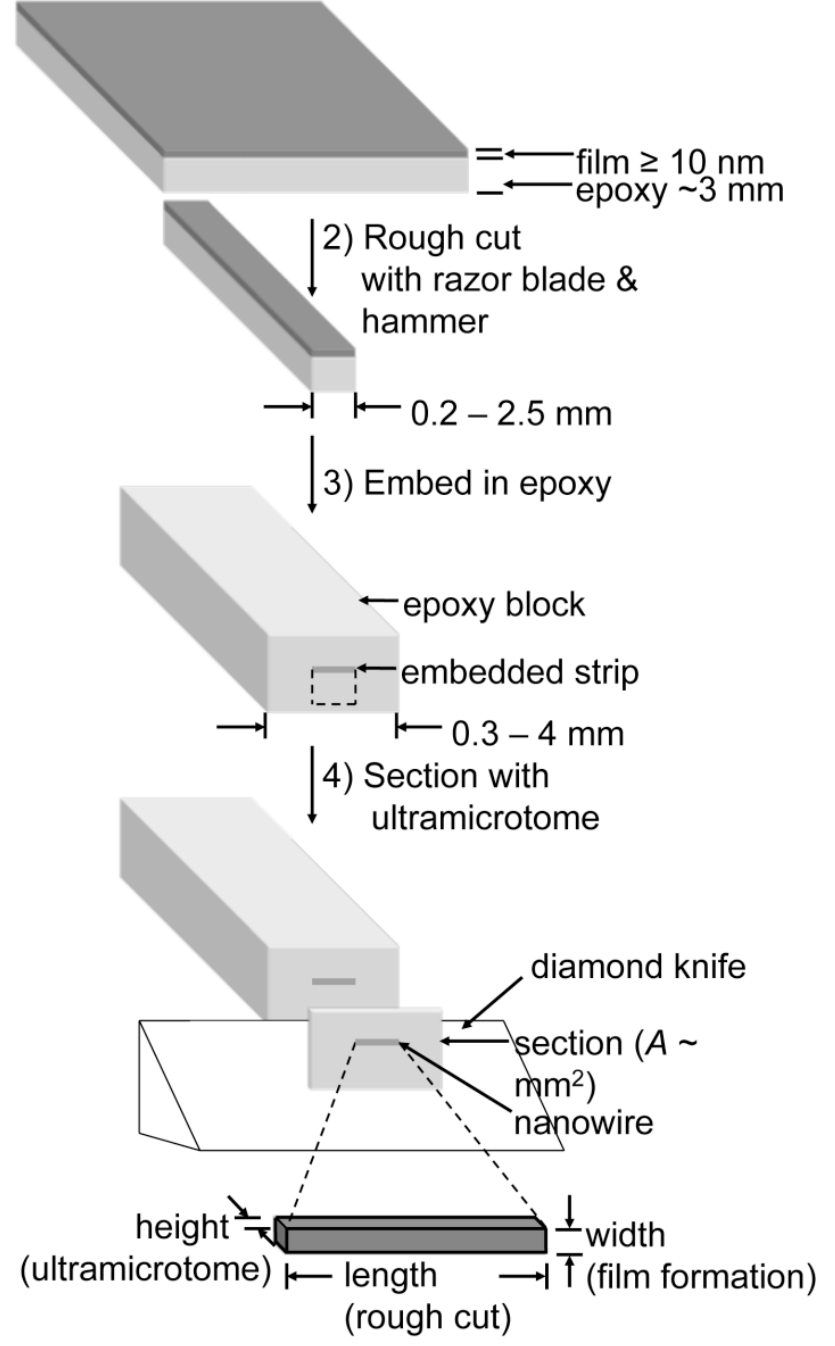

Figure 2. Summary of the process used for fabricating nanowires of loosely defined length (> $100 \mu \mathrm{m})$ by sectioning thin films. (1) A piece of flat epoxy served as the substrate for deposition of a metallic, polymeric, semiconducting, or oxide film. (2) A rough cut provided a strip of this film supported by epoxy, (3) which we embedded in additional epoxy. (4) Ultrathin sectioning (nanoskiving) and removal of the epoxy matrix formed nanowires in which each dimension was controlled by a different step of the process. Reproduced with permission from ref. [57]. Copyright 2010, American Chemical Society. 


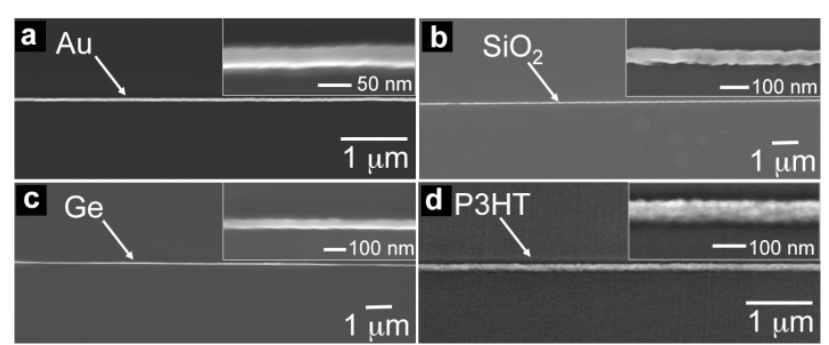

Figure 3. Examples of representative spans of nanowires of four classes of materials formed by obtaining sections of the metallic, ceramic, semiconducting, and polymeric thin films. Each nanowire is physically continuous over $>100 \mu \mathrm{m}$. Reproduced with permission from ref. [57]. Copyright 2010, American Chemical Society.

Figure 4 summarizes the results of the materials tested and makes predictions for materials we did not test or for which we did not have enough data. We assumed all metallic thin films were evaporated or sputter-coated, and thus polycrystalline. We assumed covalent solids (e.g., silicon and germanium) were evaporated, and thus amorphous. The designation "intact" indicates that the material did not fragment when sectioned. That is, these materials yielded long unbroken nanowires $(>100 \mu \mathrm{m})$ when cutting perpendicular to the edge of the embedded thin film. We applied the term "fragmented" to films that fractured extensively into segments $<10$ $\mu \mathrm{m}$. We labeled films as "borderline" as those whose rate of fragmentation depended strongly on the method of deposition, the size and geometry of the structure (e.g., nanowires or twodimensional arrays of sub-micron particles), and the orientation of the thin film with respect to the direction of cutting. We designated all other materials in the chart as either "predicted to be intact" or "predicted to be fragmented" based on the probable mechanical properties of a thin film of the material, and assumed i) that the film could be formed and ii) that it could be sectioned in an inert atmosphere. Interesting materials to which we have not yet applied nanoskiving, or that we are just starting to explore, are those with internal porous or laminated structures (e.g., graphite, montmorillonite, or block copolymers). 


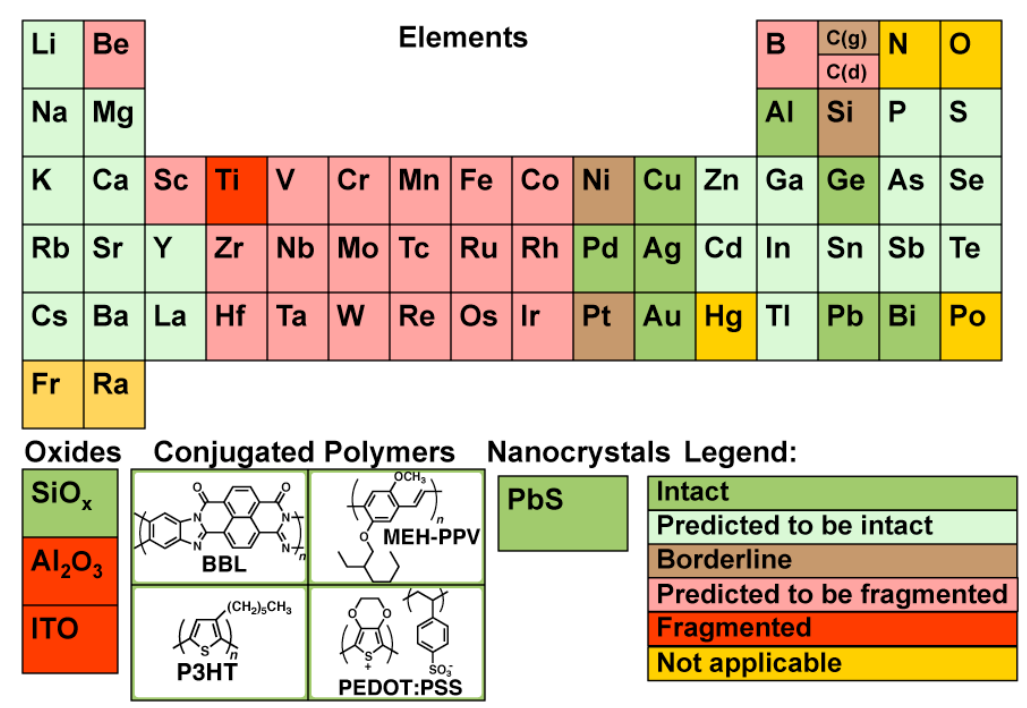

Figure 4. Summary of findings and predictions regarding the abilities of elements, oxides, polymers, and nanocrystals to form nanowires by sectioning thin films. Reproduced with permission from ref. [57]. Copyright 2010, American Chemical Society.

\subsection{Combination of Nanoskiving with Photolithography}

Patterning the thin film before embedding and sectioning can produce structures more complex than single nanowires. Figure 5 summarizes a procedure that begins with photolithographic patterning of a thin metallic film into stripes on a flat epoxy wafer. The film can be patterned first on a silicon wafer and transferred to the epoxy, ${ }^{[65]}$ or it can be patterned on the epoxy directly. ${ }^{[61]}$ Sectioning these stripes produces a collinear array of nanowires. ${ }^{[65]}$ The key result of the process is that each dimension is determined precisely: the length by photolithography, the width by the thickness of the evaporated film of gold, and the height by the set thickness of the ultramicrotome. Section 4.2 describes the optical properties of monodisperse, collinear nanowires of gold. ${ }^{[65]}$ 
1) Pattern film on flat epoxy substrate:

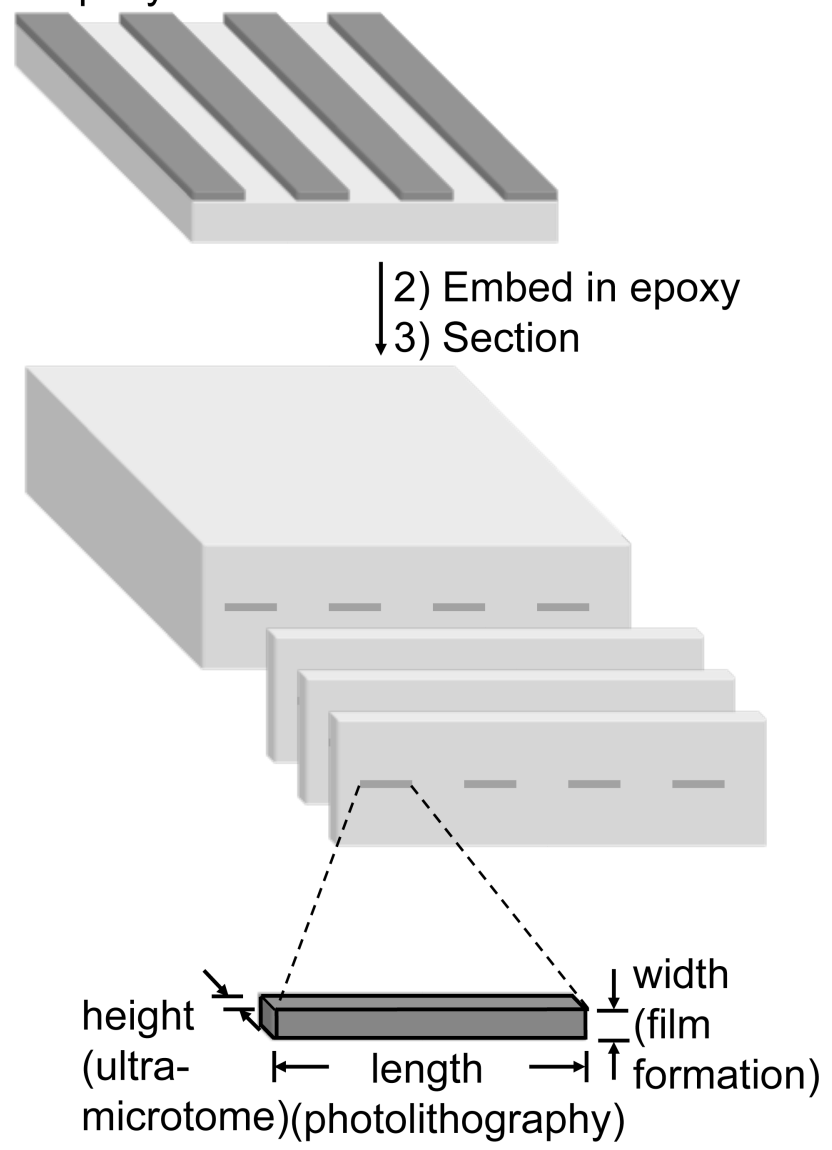

Figure 5. A modification of the procedure shown in Figure 2. (1) Photolithographic patterning produces stripes of a thin film on an epoxy substrate. (2) Embedding this substrate in more epoxy produces a block that, when sectioned (3), yields collinear arrays of nanowires embedded in thin epoxy slabs. Each dimension of the nanowire is determined by a specific part of the process.

\subsection{Combination of Nanoskiving with Soft Lithography}

Nanoskiving can produce complex, two-dimensional patterns in processes that combine soft lithographic molding (e.g. replica molding of epoxy using a PDMS mold) with thin-film deposition and nanoskiving. Figure 6a summarizes the process used to section topographically patterned thin films and depicts the two orientations — perpendicular and parallel—with which the path of cutting can intersect the film. Cutting perpendicular to a topographically patterned thin film produces a structure whose geometry is the cross sectional profile of the original 
molded structure, as shown (6b). ${ }^{[50]}$ Cutting parallel to a topographically patterned film produces parallel nanowires that correspond to the sidewalls of the topographic features of the grating (6c).

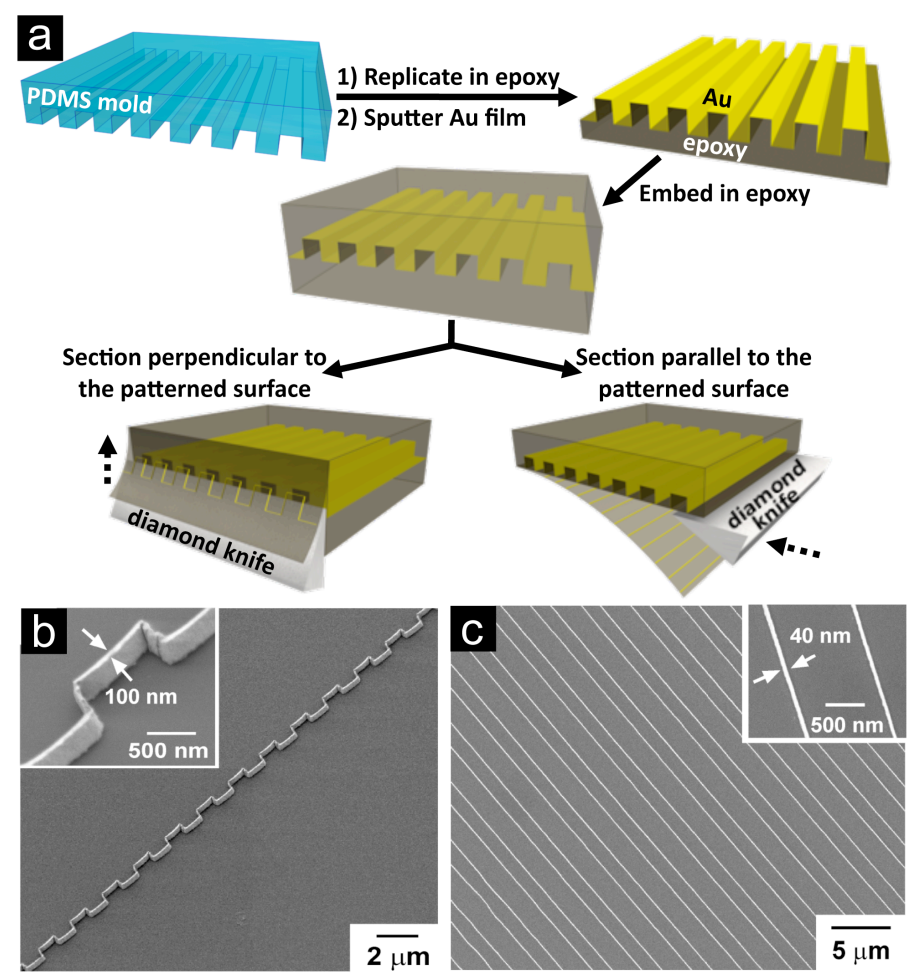

Figure 6. (a) Two general strategies to generate nanostructures by sectioning thin films coated on a polymer substrate bearing relief features. Soft lithographic procedures generate a PDMS mold, which templates the formation of an inverse replica in epoxy. Physical vapor deposition produces a metallic film (e.g., gold) on the replica. (Sputtering can be used to coat the epoxy conformally, while evaporation can be used to coat only the desired sidewalls, by line-of-sight deposition.) Additional epoxy embeds the entire structure to form a block. Sectioning the block perpendicular to the topographic features produces a nanowire with the form of a crosssectional profile of the grating (b), while sectioning parallel generates parallel nanowires (c), which come from the metalized sidewalls of the embedded structure. Reproduced with permission from ref. [50]. Copyright 2008, American Chemical Society.

\subsection{Equipment and Materials: Minimum Requirements}

Almost all of the equipment needed for nanoskiving can be found in shared facilities at most universities. The most important item needed to do nanoskiving is an ultramicrotome, 
which is standard equipment in laboratories of electron microscopy. New ultramicrotomes are approximately $\$ 60,000$. We generally deposit metallic films by evaportion or sputter-coating. Each method has useful characteristics. Electron-beam or thermal evaporation produces a collimated beam of atoms, which can coat selectively the sidewalls of photolithographic features using line-of-sight deposition. The advantage of sputter-coating is that it can coat the sidewalls of topographic features conformally. Spin-coating produces films of polymers, with uniform thickness (of a few nm to several hundred $\mathrm{nm}$ ), on planar substrates. We have also formed thin films using electroless deposition, growth in an electrochemical cell, and plasma-enhanced chemical vapor deposition (PECVD). All of these methods produce films that are either polycrystalline or amorphous that, when sectioned, produce nanostructures of roughly the same morphology (but likely retrograde due to compressive and shear stresses during sectioning) as the film from which it was cut. Shape-selective synthesis of micro- and nanoparticles of a variety of materials can produce single-crystalline precursors for nanoskiving. ${ }^{[66,67]}$ Single-crystalline gold microplates, when sectioned, yielded single-crystalline gold nanowires that behaved as lowloss plasmonic resonators, which could be used to guide light in sub-wavelength dimensions. ${ }^{[61]}$

\subsection{Scope}

This review is organized by optical and electronic applications of structures produced by nanoskiving. Electronic applications include metallic nanowires as nanoelectrodes for electrochemistry, chemoresistive nanowires of conjugated polymers and palladium, and a heterostructure of conjugated polymers, which exhibited a photovoltaic effect when placed between electrodes with different work functions. Optical applications include linear and twodimensional arrays of metallic nanostructures for applications based on localized surface 
plasmon resonances (LSPRs) for frequency-selective surfaces, and high-quality, singlecrystalline gold nanowires for plasmonic waveguiding. We also describe a method of stacking and arranging these structures with each other and with pre-deposited structures that is useful in both electronic and optical applications of nanoskiving.

\section{Electronic Applications of Nanoskiving}

\subsection{Introduction}

Conventional methods of nanofabrication are already well-suited to (and were developed for) nanoelectronic applications. ${ }^{[5]}$ Modern microelectronic devices have had nanoscale dimensions for about a decade, although the interesting effects of reductions in size in microelectronics (e.g., tunneling through leaky gate dielectrics) are usually treated as something to be suppressed, rather than something to be exploited. ${ }^{[68]}$ True nanoelectronics is in an exploratory phase. It combines new materials and structures—carbon nanotubes, ${ }^{[3]}$ graphene, ${ }^{[69]}$ and semiconducting, metallic, or dielectric nanowires ${ }^{[70]}$ — that are addressed using conventional lithography. Some of the most exciting new directions include integrating nanoelectronic and nanophotonics components on the same chip. ${ }^{[71]}$

This section highlights five applications of nanoskiving to which conventional methods of fabrication are not easily applied. The structures and applications are fabrication of i) electrically isolated, patterned electrodes for electrochemistry; ${ }^{[72]}$ ii) individually addressable, parallel nanowires separated by a nanogap for nanoelectrodes; ${ }^{[73]}$ iii) parallel nanowires of metals ${ }^{[74]}$ and polymers ${ }^{[75]}$ with high pitch for chemical sensing; iv) junctions of nanowires positioned using magnetic interactions for different purposes; ${ }^{[74]}$ and v) heterostructures of conjugated polymers for photodetectors. ${ }^{[62]}$ 


\subsection{Fabrication of Nanoelectrodes for Electrodeposition}

One of the challenges in using patterned nanoelectrodes for electrochemistry is connecting the nanostructures to an electrometer in a way that blocks the conductive substrate from the solution, so that only the patterned nanostructures are exposed to the electrochemical solution. In the first report of nanoskiving, Xu et al. fabricated an array of metallic nanowires embedded in an epoxy slab and placed it on a substrate bearing a gold film. ${ }^{[72]}$ The gold film was in contact with the underside of the metallic nanostructures. The epoxy slab covered the conductive substrate, so that only the surfaces of the gold nanowires were exposed. The gold substrate, epoxy slab, and embedded nanostructures were immersed in a solution for the electrochemical deposition of gold. The top surfaces of the nanowires were the only facets of the nanowires exposed to the electrochemical solution, and thus were the only facets plated with gold. This experiment was the first demonstration of the electrical continuity of nanowires fabricated by nanoskiving. ${ }^{[72]}$

\subsection{Fabrication of Addressable Nanowires Separated by a Nanogap}

Parallel nanowires that are separated by a nanoscale gap in the lateral dimension are useful for a number of applications, in sensing, ${ }^{[76,77]}$ as electrodes for dielectrophoresis ${ }^{[78,79]}$ and

electrochemistry, ${ }^{[80]}$ in molecular electronics, ${ }^{[81,82]}$ and as a platform for interrogating phenomena that occur over the nanoscale in charge transport ${ }^{[35]}$ or plasmonics. ${ }^{[83]}$ There are few methods of generating such nanowire electrodes. Electron-beam lithography and FIB lithography and milling are two such methods, but, in addition to the usual drawbacks of high cost, inconvenience, and low throughput, it is difficult to produce nanoscale gaps over large lengths. It 
is nearly impossible in an academic laboratory to use photolithography to contact structures that are separated by less than $100 \mathrm{~nm}$. A simple method to produce electrodes bearing a nanogap could be a tool of significance in discovery-driven research in nanoelectronics.

We fabricated transferrable, parallel electrodes bearing a nanogap using a combination of micromolding in capillaries, physical vapor deposition, and nanoskiving. Figure 7a is a schematic drawing of three parallel nanowires that are separated by gaps $<100 \mathrm{~nm}$ in the parallel region and $>10 \mu \mathrm{m}$ in the diverging, addressable region. ${ }^{[73]}$ We demonstrated the electrical continuity of these nanowires by electrodepositing the conducting polymer, polyaniline (PANI), in the nanogap between two nanowires. We addressed the nanowires individually using lowresolution $(>10 \mu \mathrm{m})$ photolithography using a printed transparency mask in contact mode. The two nanowires together served as the working electrode for the deposition of PANI. Figures $\mathbf{7 b}$ and 7c show two parallel nanowires, separated by a gap of $30 \mathrm{~nm}$, before and after electrodeposition. The gap between the nanowires was conductive when spanned by the PANI fibers, and insulating before the fibers were deposited and after they were etched. ${ }^{[73]}$ 

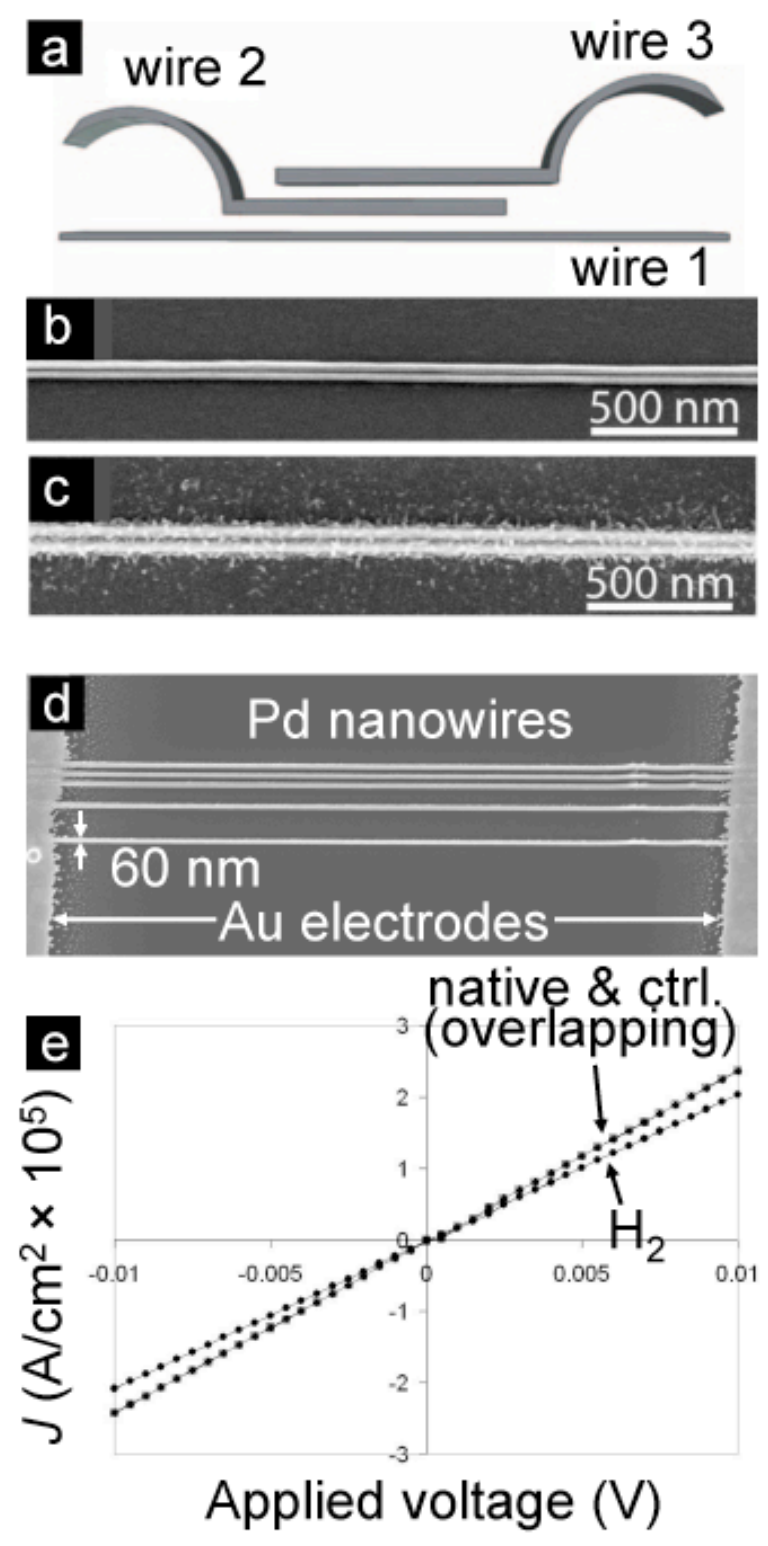

Figure 7. (a) A schematic drawing of three parallel, addressable gold nanowires. (b) Parallel gold nanowires separated by a $30 \mathrm{~nm}$ gap. (c) The same gold nanowires after electrochemical deposition of polyaniline. Reproduced with permission from ref. [73]. Copyright 2008, American Chemical Society. (d) A group of five parallel nanowires of palladium spanning a 10- $\mu$ gap between Au electrodes. (e) The electrical response of the nanowires in their native state, when exposed to a stream of hydrogen gas, and a control experiment in which the nanowire was exposed to a stream of compressed air.

\subsection{Fabrication of Chemoresistive Nanowires of Palladium}

Nanowires may have properties useful in sensing, because they have a high ratio of surface area to volume; this feature permits rapid diffusion of an analyte into and out of a wire 
(or adsorption/desorption from its surface), ${ }^{[84,85]}$ a strong influence of adsorbed material on properties such as conductivity, and rates of response and recovery that are superior to those of devices based on thin films or fibrous networks. Palladium is an example of a material having properties useful in sensing because of its resistance to oxidation and reproducible loss in conductivity (chemoresistivity) upon absorption of hydrogen. (It is also soft enough to be sectioned with the ultramicrotome without fragmentation). Penner and coworkers have fabricated and studied palladium nanowires and their characteristics as hydrogen gas sensors. ${ }^{[86]}$ These nanowires can be prepared by templated electrodeposition, ${ }^{[77]}$ or by step-edge decoration of highly oriented pyrolytic graphite, or other templates. ${ }^{[87]}$ We have fabricated palladium nanowires with rectangular cross sections and high pitch using a sequence of two steps: iterative template stripping, ${ }^{[88]}$ followed by nanoskiving. ${ }^{[74]}$

To characterize the nanowires electrically, we tested them for function as sensors for hydrogen gas. We began by placing an epoxy slab bearing five palladium nanowires $(w=60 \mathrm{~nm}$, $h=80 \mathrm{~nm}$ ) on an insulating substrate. We then deposited gold electrodes through a stencil mask to define a span of $10 \mu \mathrm{m}$ (Figure 7d). Etching the epoxy matrix freed the sides of the nanowires. Figure 7e shows three plots of current density vs. applied voltage. The first, "native", represents the conductivity of the nanowires in the ambient atmosphere of the laboratory. The second, " $\mathrm{H}_{2}$ ", represents the lower conductivity of the nanowire when exposed to a stream of hydrogen gas. The third, "ctrl", is a control experiment, in which we exposed the nanowires to a stream of air. The control experiment and the native conductivity of the nanowires exhibited identical electrical characteristics. From the value of current at $10 \mathrm{mV}$ and the dimensions of the nanowires, we calculated the conductivity of the nanowires to be $2.4 \times 10^{4} \Omega^{-1} \mathrm{~cm}^{-1}$. For comparison, the conductivity of bulk palladium is $9.5 \times 10^{4} \Omega^{-1} \mathrm{~cm}^{-1}$. 


\subsection{Fabrication of Chemoresistive Conjugated Polymer Nanowires}

Organic semiconductors are a class of materials whose properties (e.g., reversible behavior in cycles of oxidation and reduction, and modifiable conductivity by electrical gating) render them potentially attractive for chemical and biological sensing ${ }^{[89]}$ Incorporation of molecular recognition elements into semiconducting, conjugated polymer nanowires is relatively straightforward by synthesis, while modifications of carbon nanotubes and inorganic nanowires require functionalization of the surfaces, carried out post-fabrication. ${ }^{[90]}$ Other possible uses for conjugated polymer nanowires are as tools for studying one-dimensional charge transport, ${ }^{[91]}$ or as field-effect transistors, ${ }^{[92]}$ actuators ${ }^{[93]}$ or interconnects. ${ }^{[94]}$

There is not yet a truly general technique for the fabrication of conjugated polymer nanowires. Examples of methods that satisfy some of the criteria of cost, accessibility, and applicability to different materials, are electrodeposition in templates, ${ }^{[77]}$ dip-pen nanolithography, ${ }^{[44]}$ nanoimprint lithography, ${ }^{[95]}$ and electrospinning ${ }^{[96]}$ —a well-studied technique that can produce mats or aligned collections of nanowires ${ }^{[96]}$ Craighead and coworkers have used scanned electrospinning ${ }^{[97]}$ to deposit single nanowires of polyaniline ${ }^{[98]}$ and poly(3-hexylthiophene $)^{[92]}$ on a rotating substrate, while Xia and coworkers have developed an approach to deposit uniaxial collections of nanofibers of a range of inorganic and organic materials. ${ }^{[99,100]}$

Using a procedure that involves stacking spin-coated films of conjugated polymers, followed by nanoskiving, we have generated nanowires with rectangular cross sections individually, in bundles, or in parallel, with high pitch. ${ }^{[75]}$ We began by spin-coating two conjugated polymers, poly(benizimidazo-benzophenantrholine) ladder (BBL) and poly(2- 
methoxy-5-(2'-ethylhexyloxy)-1,4-phenylenevinylene) (MEH-PPV) alternately on the same substrate, such that fifty 100 -nm-thick layers of BBL were separated by fifty 100 -nm-thick layers of MEH-PPV. Release of this free-standing, 10- $\mu \mathrm{m}$-thick film, and subsequent sectioning perpendicular to the plane of the layers, provided a cross section which bore 100-nm-wide strips of the two conjugated polymers. Etching the MEH-PPV with an air plasma left behind parallel BBL nanowires (Figures 8a and 8b), and dissolving BBL in methanesulfonic acid left behind MEH-PPV nanowires (Figures 8c and 8d). Figure 8e is a plot of current density vs. voltage ( $J$ $V)$ of a group of MEH-PPV nanowires, of the type shown in Figure 8d, when exposed to $\mathrm{I}_{2}$ vapor.
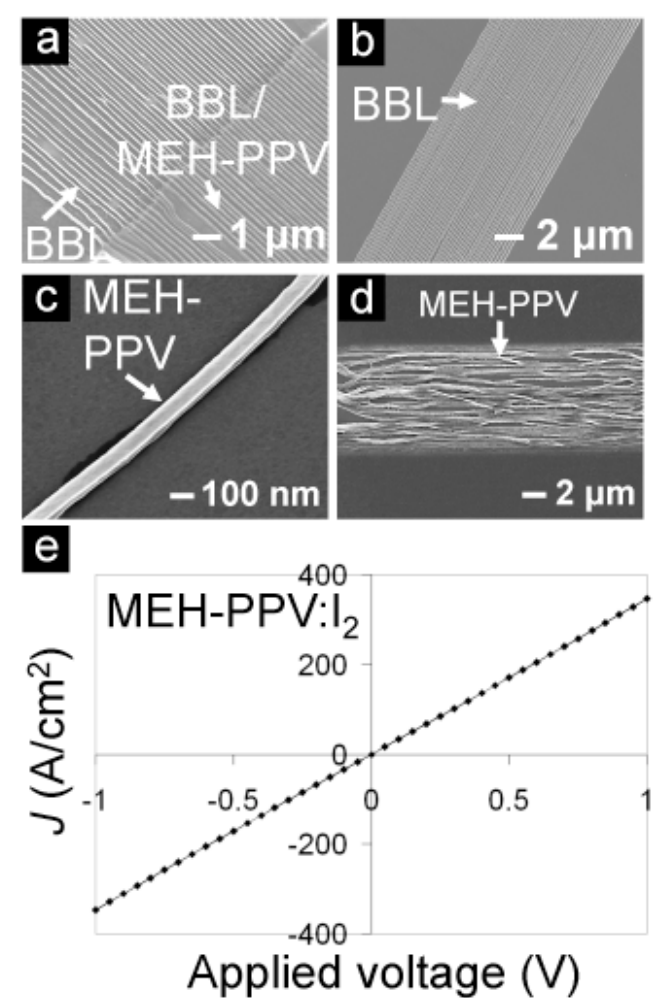

Figure 8. (a) BBL nanowires before (bottom right) and after (top left) etching the sacrificial polymer, MEH-PPV, with an air plasma. The unetched region was protected with a conformal slab of PDMS. (b) A group of 50 parallel BBL nanowires with $200 \mathrm{~nm}$ pitch. (c) A single $M E H$ $P P V$ nanowire with a square cross section of $100 \mathrm{~nm} \times 100 \mathrm{~nm}$. (d) A group of fifty MEH-PPV nanowires. (e) A J-V plot of the MEH-PPV nanowires in (d) doped by iodine $\left(I_{2}\right)$ vapor. Reproduced with permission from ref. [75]. Copyright 2008, American Chemical Society. 


\subsection{Arranging Nanowires of Different Types Using Magnetic Mooring}

One of the central challenges in promoting discoveries of nanoscience into technological applications is the ability to manipulate and position nanostructures on a surface. We refer specifically to nanostructures fabricated by bottom-up methods, such as solution-phase synthesis $^{[66]}$ or vapor-liquid-solid growth ${ }^{[70]}$ (structures fabricated by top-down procedures are usually formed where the template provided by the photomask dictates). The usual procedure to interrogate a nanostructure prepared by bottom-up synthesis is to deposit structures randomly, and then to select a serendipitously positioned structure- for example, a nanowire spanning two electrodes, or situated in the proper orientation on an optical waveguide. The more elements that make up a system - e.g., nanowires, waveguides, electrodes, and quantum dots - the lower the probability that random assembly can generate a useful geometry. This section focuses on onedimensional structures ${ }^{[3]}$ but the processes we describe would be applicable to other structures as well.

There are several methods of aligning nanowires in groups and individually. Methods to align nanowires in group include shear alignment of nanowires suspended in fluids, ${ }^{[101-105]}$ including wafer-scale alignment in bubble-blown films; ${ }^{[106]}$ brushing suspensions of nanowires over a lithographically patterned substrate to create highly aligned regions of nanowires on exposed areas; $;{ }^{[107]}$ and alignment of nanowires in a Langmuir-Blodgett trough. ${ }^{[108]}$ Methods of positioning single nanowires include optical tweezing ${ }^{[109]}$ and opto-electronic tweezing; ${ }^{[110]}$ methods of manipulation by direct contact with scanning probe tips ${ }^{[111]}$ and micromanipulators ${ }^{[112]}$ and electrophoretic alignment of over pre-patterned electrodes. ${ }^{[113]}$

In nanoskiving, the generation of the thin slabs-usually removed in the final step of fabrication - is one of the most useful characteristics of the technique. The slabs are visible, 
manipulable objects that can be overlayed—along with the nanostructures they contain — with structures on the substrate. Complex structures embedded in the polymer slab retain their orientation during manipulations of the slab. For structures formed by laying slabs on top of one another, we have developed a procedure that combines nanoskiving with non-contact, magnetic manipulation of the slabs ("magnetic mooring", Figure 9). The key step in magnetic mooring is co-embedding ferromagnetic particles (we used nickel films or powder) with the metallic or polymeric nanostructures to be positioned. In an initial demonstration of magnetic mooring, we transferred slabs containing nickel particles and gold nanowires to a substrate, along with $\sim 5 \mu \mathrm{L}$ of water. The slabs floated on the surface of a drop of water, and were thus mobile under the influence of an external permanent magnet attached to a micromanipulator (Figure 9a). As the water evaporated, the slabs, along with the nanostructures they contained, deposited on (and adhered to) the substrate, with an average deviation from the intended position of $16 \mu \mathrm{m}$. This registration is crude, but useful in some applications. Figures $9 \mathbf{b}$ and $9 \mathbf{c}$ show crossing singlecrystalline and polycrystalline gold nanowires. 


\section{a}

1) Deposit Ni (left) and Au (right) films on flat epoxy substrates:

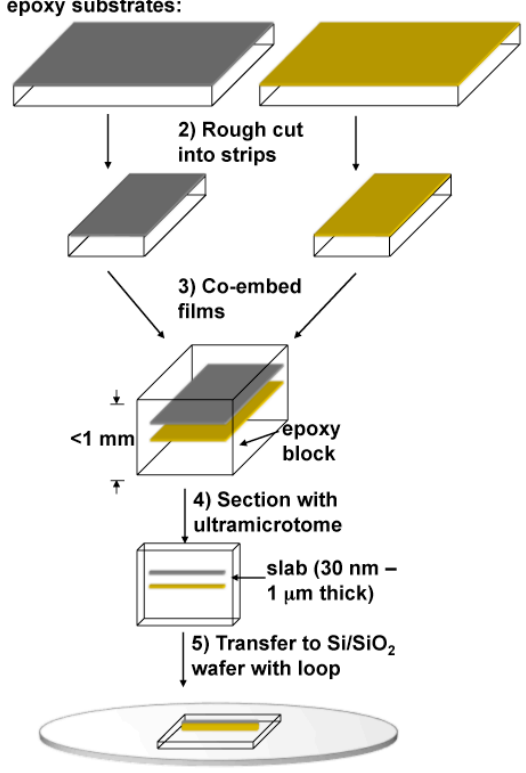

Il. Magnetic Mooring

6) (Optional) Etch Ni and epoxy:

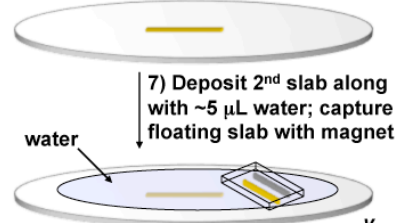
$\left.\begin{array}{l}\text { cylindrical magnets } \\ \text { (on movable stage) }\end{array}\left|\begin{array}{l}\mathrm{N} \\ \mathrm{S} \mid \mathrm{S}\end{array}\right|\right\}+\overbrace{\theta}^{y} x$

8) Guide floating slab to fixed nanowire
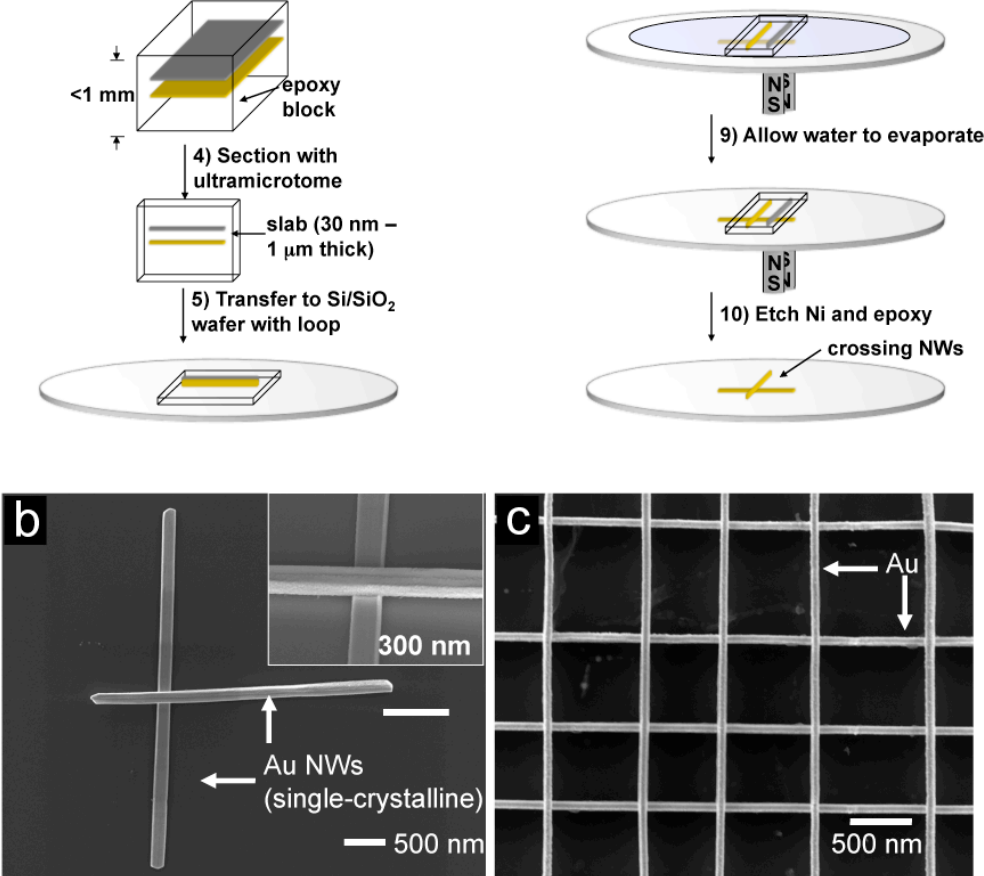

Figure 9. (a) Schematic representations of the methods used for fabrication (I. Nanoskiving) and positioning (II. Magnetic Mooring) of nanowires. Single-crystalline (b) and polycrystalline (c) gold nanowires fabricated using nanoskiving and positioned using magnetic mooring. Reproduced with permission from ref. [74]. Copyright 2009, American Chemical Society.

To show that it was possible to form electrically continuous junctions between nanowires of different types, we placed a single nanowire of poly(3-hexylthiophene) perpendicularly across the gap between two parallel gold nanowires. This geometry could be useful in measuring nanoscale charge transport in optoelectronic polymers, and in the fabrication of chemical sensors ${ }^{[14]}$ or field-effect transistors based on single nanowires. ${ }^{[92]}$ We deposited two parallel gold nanowires, which were embedded in the same epoxy slab. Separately, we fabricated a poly(3-hexylthiophene) nanowire $(100 \mathrm{~nm} \times 100 \mathrm{~nm}$ cross section), co-embedded with nickel 
powder in epoxy, positioned it to span the $50-\mu \mathrm{m}$ gap between gold nanowires. Poly(3-

hexylthiophene) undergoes an insulator-to-metal transition upon exposure to $\mathrm{I}_{2} \cdot{ }^{[115]}$ Electrical measurements of the polymer nanowire—using the gold nanowires as electrodes—could detect the presence or absence of a solid piece of $\mathrm{I}_{2}$ placed in the vicinity of the nanowire. It should also be possible to use this technique for four-terminal measurements, which would allow decoupling of the contact resistance from the true resistance of a nanowire. ${ }^{[84]}$

\subsection{Fabrication of an Ordered Bulk Heterojunction of Conjugated Polymers}

Nanoskiving is one of a few techniques for nanoscale patterning in which features can be made of different materials, and in which components fabricated in different materials touch in the lateral dimension. Forming densely packed features that touch laterally may be able to address a long-standing problem in organic photovoltaic cells: that is, to fabricate a structure in which two organic semiconductors (electron-donating and electron-accepting) are laterally separated on the scale of exciton diffusion, or approximately $10 \mathrm{~nm} \cdot{ }^{[116]}$ A persistent challenge in the fabrication of organic photovoltaic devices is that the distance an exciton can travel before it decays (the exciton diffusion length, or $L_{D}$ ) is about 10 times shorter than the thickness of material required for efficient absorption of photons (100 to $200 \mathrm{~nm}$ ). The architecture that satisfies the requirement that both $L_{D}$ and the thickness for optimal absorption of light is known as the ordered bulk heterojunction. ${ }^{[117]}$ It has a cross section of electron-donating and electronaccepting phases that is intermixed on the length scale of $L_{D}$ and is 100 to $200 \mathrm{~nm}$ thick.

We used nanoskiving to fabricate an ordered bulk heterojunction of two conjugated polymers. The process had three steps: i) spin-coating a composite film with 100 alternating layers of BBL (e-acceptor) and MEH-PPV (e-donor); ii) rolling this multilayer film into a 
cylinder (a "jelly roll”); and iii) nanoskiving the jelly roll. ${ }^{[62]}$ The cross-section of a slab of the jelly roll had an interdigitated arrangement of the two polymers. The thickness of the slab was determined by the ultramicrotome and the spacing between the two materials was determined by the details of the spin-coating. We placed a slice of the jelly roll between two electrodes with different work functions (Figure 10a), tin-doped indium oxide (ITO) and eutectic galliumindium (EGaIn), and observed a photovoltaic response when we irradiated the stack with white light (10b). Selective excitation of BBL (which does not produce a photovoltaic response by itself) with red light (which MEH-PPV does not absorb) confirmed that the photovoltaic effect was the result of photoinduced charge transfer between BBL and MEH-PPV. Although the power conversion efficiency of these structures were low $(<0.1 \%)$, we believe that this approach to fabricating donor/acceptor heterojunctions could be useful in photophysical studies, and might ultimately suggest new approaches to OPV devices. ${ }^{[62]}$ 

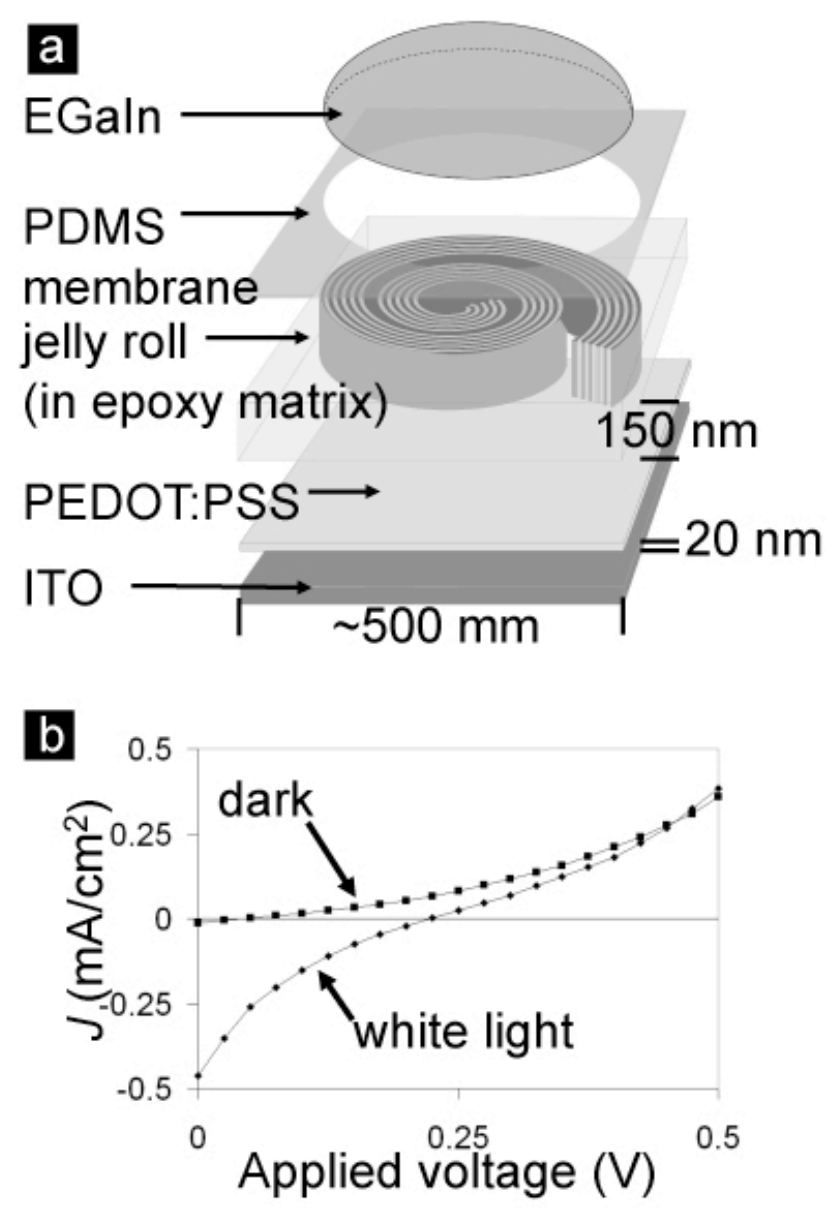

Figure 10. (a) A schematic drawing of a heterojunction ("jelly roll") of conjugated polymers sandwiched between two electrodes with asymmetric work functions. (b) Upon irradiation with white light, the junction produced a photovoltaic effect. Reproduced with permission from ref. [62]. Copyright 2008, Wiley-VCH GmbH \& Co. KGaA.

\section{Optical Applications of Nanoskiving}

\subsection{Introduction}

Metallic nanostructures with well-defined nano and microscale geometries are the building blocks of the branch of optics known as plasmonics. ${ }^{[118,119]}$ A surface plasmon is a quantum of oscillation of charge at a metal-dielectric interface, driven by electromagnetic radiation. Localized surface plasmon resonances (LSPRs) can be excited in nanoparticles whose dimensions are much smaller than the wavelength of excitation. The energy of the LSPR is a function of the size and shape of the particle, and its dielectric environment. ${ }^{[118]}$ Applications of 
plasmonic nanoparticles include optical filters, ${ }^{[120,121]}$ substrates for optical detection of chemical and biological analytes using LSPRs ${ }^{[122]}$ or surface-enhanced Raman scattering (SERS); ${ }^{[123-125]}$ substrates for enhanced luminosity ${ }^{[126]}$ materials to augment absorption in thin-film photovoltaic devices; ${ }^{[127]}$ metamaterials ${ }^{[7,128]}$ with negative magnetic permeabilities ${ }^{[129]}$ and refractive indices ${ }^{[130]}$ and materials for perfect lenses, ${ }^{[131]}$ and invisibility cloaking. ${ }^{[132]}$

The most sophisticated arrays of plasmonic structures are fabricated using EBL, ${ }^{[133]}$ FIB, ${ }^{[130]}$ or direct laser writing. ${ }^{[134]}$ There are also a number of chemical, soft lithographic, and other unconventional approaches to producing plasmonic materials. ${ }^{[119]}$ Solution-phase synthesis can produce single-crystalline metallic structures of different shapes and materials. ${ }^{[66,135]}$ Nanosphere lithography, pioneered by Van Duyne and coworkers, uses self-assembled spheres as a stencil mask, in which the void spaces between the spheres direct the deposition of metal on the substrate by evaporation. ${ }^{[136,137]}$ Rogers, Odom, Nuzzo, and coworkers have used soft lithographic techniques, such as patterning photoresists with conformal phase-shifting masks, ${ }^{[138]}$ as well as soft nanoimprint lithography, ${ }^{[139]}$ to form arrays of nanoholes in metallic films ${ }^{[140]}$ and pyramidal shells. ${ }^{[141]}$ This section describes the use of nanoskiving to generate nanostructures for a variety of optical applications.

\subsection{Fabrication of Gold Nanowires and Size-Dependent Surface Plasmon Resonance}

The combination of patterning or molding, thin-film deposition, and sectioning can control each dimension of the structures produced by nanoskiving. ${ }^{[50]}$ Plasmonic applicationse.g., sensors based on changes in the frequency of LSPRs, optical polarizers, filters - require uniform absorption across arrays of particles. Monodisperse particles satisfy this requirement, while polydisperse particles absorb broadly. ${ }^{[2]}$ Figure 5 summarizes a method to fabricate 
collinear arrays of optically indistinguishable nanowires of gold that uses nanoskiving. ${ }^{[65]}$ The cross sections of the nanowires had dimensions as small as $10 \mathrm{~nm} \times 30 \mathrm{~nm}$; all nanowires were 2 $\mu \mathrm{m}$ long.

Illumination of groups of these nanowires excited plasmon resonances along their transverse axes. In order to test the optical homogeneity of the nanowires, Xu et al. collected the spectra of four nanowires individually. The nanowires exhibited overlapping spectra of scattered light, which implied that they were geometrically monodisperse. There was a red shift in the peak of the scattered intensity with increasing height. This observation was consistent with finite-difference time-domain (FDTD) simulations. The ability to tune the size, shape, and composition of metallic structures is a useful capability of nanoskiving for optical applications. $^{[65]}$

\subsection{Fabrication of Single-Crystalline Gold Nanowires for Plasmonic Waveguiding}

Nanophotonic devices, including photonic integrated circuits, require waveguiding of optical energy in sub-wavelength dimensions. ${ }^{[7,142]}$ Patterned metal strips or can guide light using surface plasmon polaritons (SPPs) that propagate along a metal-dielectric interface, but efforts to produce efficient plasmonic waveguides from these structures have been hindered by the rough surfaces of polycrystalline evaporated films, which are unsuitable to support propagation of the

lengths needed. ${ }^{[143]}$ Recently, Ditlbacher and coworkers, ${ }^{[144]}$ and others, ${ }^{[145]}$ have shown that high quality, single-crystalline silver nanowires can confine the energies of incident photons to propagating SPPs, which travel along the longitudinal axes of the nanowires (in Section 4.2, LSPRs were excited along the transverse axes). For example, microfabricated strips of silver exhibited propagation lengths of $2.5 \mu \mathrm{m}$, where single crystalline silver nanowires have 
propagation lengths of $10 \mu \mathrm{m}$, due to the low losses at the smooth surface ${ }^{[144]}$ Gold nanowires might be superior to silver nanowires for practical applications, because gold is stable in air. Studies of SPPs along gold nanowires had not been performed, however, in part because the synthesis of silver nanowires was well established. ${ }^{[146]}$

Using a procedure that combined chemical synthesis of gold microplates ${ }^{[147]}$ and nanoskiving (Figure 11a), we were able to produce collinear arrays of high quality, single crystalline nanowires. ${ }^{[61]}$ Figure 11b shows a dark-field optical image and 12c shows and SEM image. In order to determine if these nanowires could be used to confine and guide light using SPPs, we mounted a nanowire on a prism and illuminated it with unpolarized white light under total internal reflection. We oriented the nanowire parallel to the evanescent wave generated by at the surface of the prism (11d). We observed light scattering from both ends ("input" and "output") of the nanowire; Figure 11e shows the spectra of wavelengths scattered from each end. The minima of the spectrum scattered from the input tip, and the maxima of the spectrum scattered from the output tip, corresponded to wavelengths at which maximum coupling of the light into the nanowire occurred due to constructive interference of the SPP modes reflecting between the two tips. These wavelengths correspond to those that reproduced themselves after a full round trip. The light did not scatter from the center of the nanowire because the wave vector of the surface plasmon is higher than that of light in air. ${ }^{[61]}$ 
a

1. Deposit gold microplates from solution onto epoxy

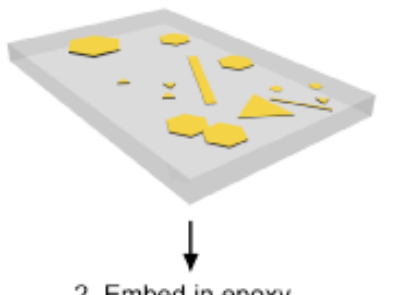

2. Embed in epoxy

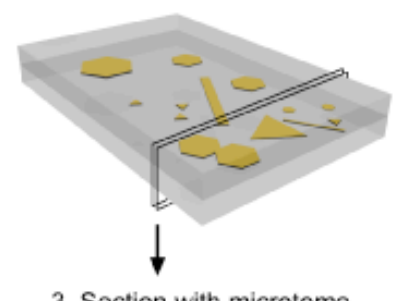

3. Section with microtome

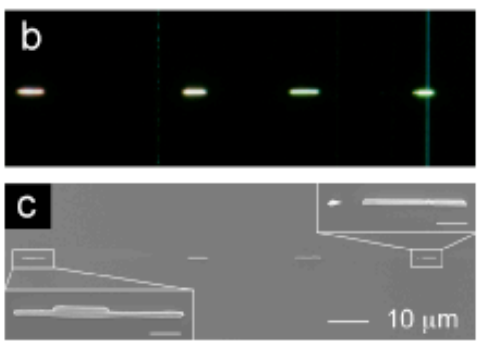

d Spectrometer

Lens

Objective
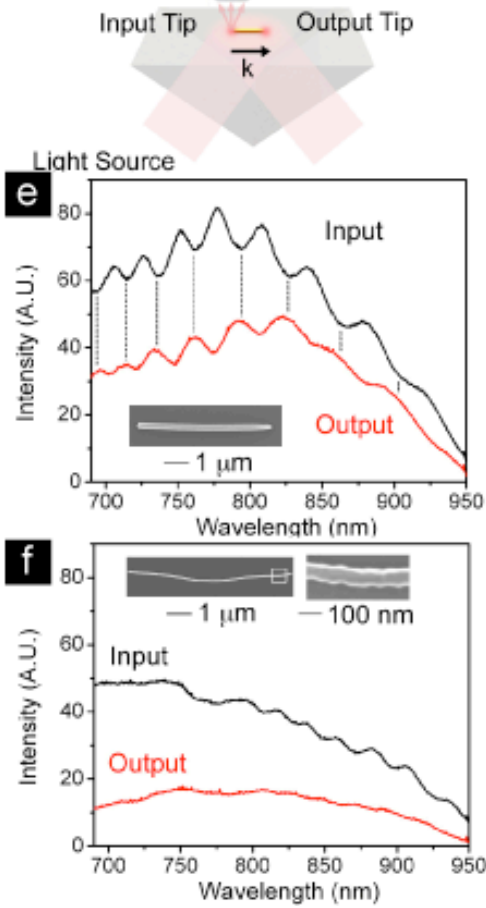

Figure 11. (a) Schematic representation of the procedure used to deposit, embed, and section gold microplates into nanowires. (b) Dark-field and (c) SEM images of a group of colinear single-crystalline nanowires. (d) Schematic drawing of the orientation of the nanowire on a prism with respect to the wave vector ( $k$ ) of the impinging white light. (e) Spectra of scattered light from both the input and output tips of the nanowires. The minima of the input and the maxima of the output intensities correspond to the wavelengths of maximum coupling into the nanowire (Fabry-Perot resonance). (f) Scattering spectra of a polycrystalline nanowire fabricated by photolithography, evaporation, and nanoskiving. The spectra do not exhibit evidence of propagating resonator modes. (g) Two nanowires placed perpendicular to each other. (h) An optical micrograph of a nanowire placed on a microfabricated waveguide. The inset is an SEM close-up. Reproduced with permission from ref. [61]. Copyright 2008, American Chemical Society.

These single-crystalline plasmonic waveguides could be stacked on top of each other, or positioned on microfabricated polymeric waveguides, using magnetic mooring, as described in section 3.6. ${ }^{[74]}$ This process could be used to generate more complex arrangements of elements in order to produce multicomponent photonic devices comprising, for example, photonic and plasmonic waveguides, ${ }^{[142]}$ semiconductor nanowires, ${ }^{[71]}$ and single-photon emitters. ${ }^{[7]}$ 


\subsection{Fabrication of 2D Arrays of Nanostructures}

Sections 4.2 and 4.3 focused on one-dimensional nanostructures. Applications such as optical filters, ${ }^{[120,121]}$ substrates for surface-enhanced Raman spectroscopy, ${ }^{[8,123]}$ and metamaterials $^{[128,130,133,137]}$ required two-dimensional arrays of nanostructures. Using a procedure that combined replica molding of a nanostructured template (for example, a set of nanorods) in epoxy by soft lithography, thin-film deposition of metal onto those rods, embedding, and sectioning parallel to the plane supporting the nanorods, we produced two-dimensional arrays of nanostructures using nanoskiving. Figure 12 outlines an example. First, we formed an array of epoxy nanoposts by soft lithographic molding. This array was coated conformally with gold by sputter coating, then coated by polypyrrole (PPy) using electrochemical growth, then coated a second time with gold by sputter coating. These procedures produced an array of three-layered, coaxial nanoposts with radial symmetry. When these composite posts were embedded in epoxy and sectioned into slabs, the slabs comprised an array of features with radial symmetry: discs of epoxy, and concentric rings of gold, PPy, and gold. These arrays could be transferred to essentially any substrate. An optional step was to remove the organic components by etching using an air plasma. Etching left behind arrays of free-standing, concentric rings of gold. Figures 13a $-13 \mathbf{h}$ show a series of structures fabricated by this and related procedures. 


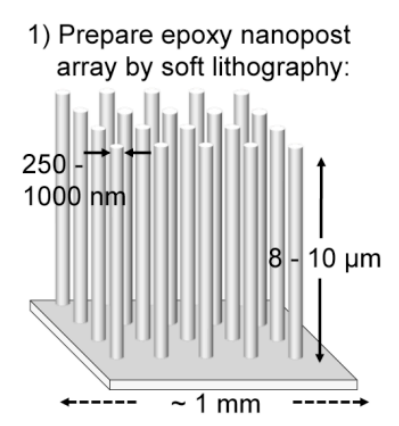

2) Deposit thin metallic film
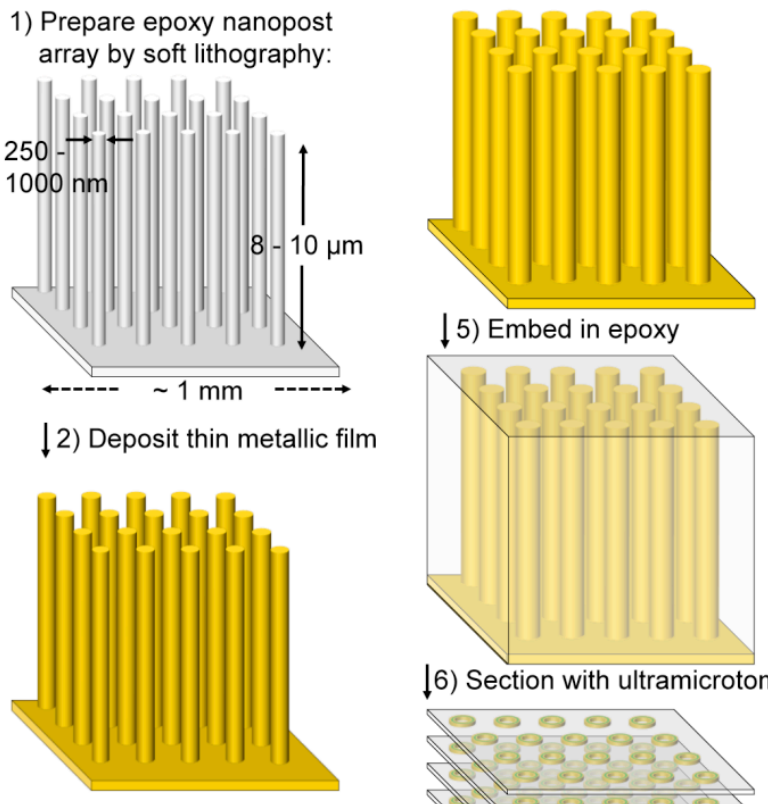

15) Embed in epoxy

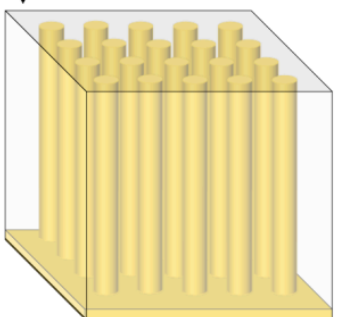

16) Section with ultramicrotome

13) Electrodeposit polypyrrole
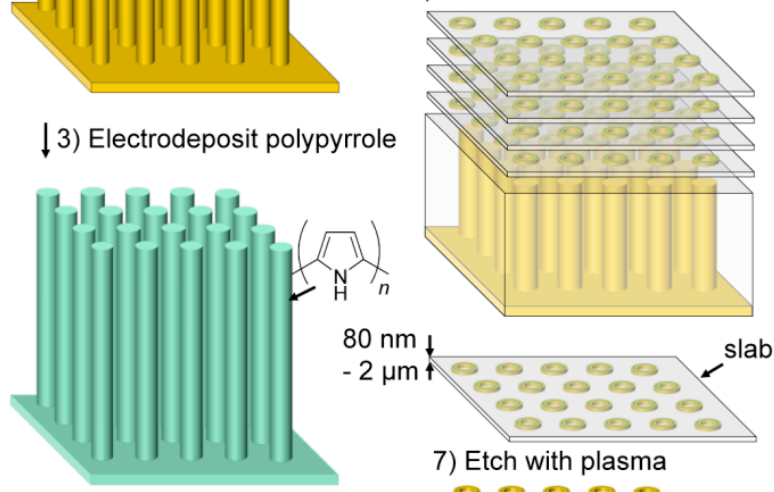

7) Etch with plasma

14) Deposit thin gold film

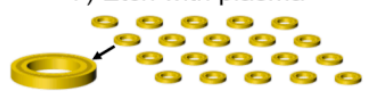

Figure 12. Summary of the procedure used to fabricate concentric rings by thin film deposition and thin sectioning of high-aspect-ratio nanoposts. Sputter-coating produced a film of Au on an array of epoxy nanoposts (step 1). This film served as the working electrode for the conformal electrodeposition of PPy (step 2). A second sputter-coating provided a nanopost array with a core-shell-core-shell composition (step 3). Embedding this structure in additional epoxy formed a block (step 4). Sectioning this block with the ultramicrotome yielded an epoxy slab containing the nanostructures (step 5). This structure could be transferred from the water bath on which the nanostructures float to any substrate (not shown). Treatment with an air plasma simultaneously etched the epoxy matrix and the PPy in between the Au rings (step 6). Reproduced with permission from ref. [59]. Copyright 2010, American Chemical Society. 

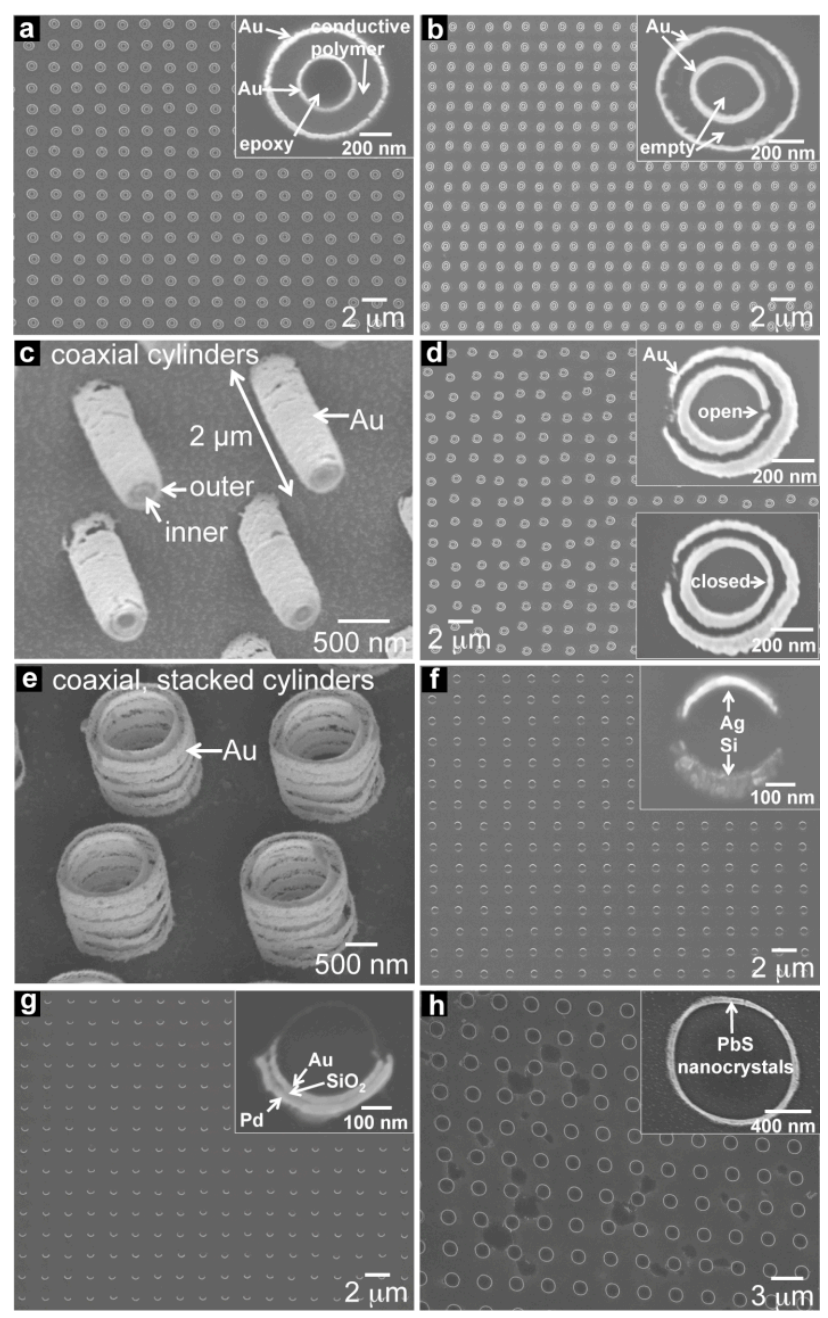

Figure 13. Scanning electron micrographs of two-dimensional arrays of nanostructures. (a) Double rings of gold separated by a layer of polypyrrole. (b) Double rings of gold after etching the organic components with an air plasma. (c) Coaxial cylinders of gold obtained by cutting a 2- $\mu m$-thick slab of the sample like that from which (b) was derived. (d) Counterfacing, concentric rings of gold. The array contains a mixture of the two structures shown in the inset. (e) Concentric, stacked, cylinders of gold. The cylinders are segmented because the sidewalls of the silicon master were scalloped due to the Bosch process of deep reactive-ion etching. (f) Counterfacing crescents of silver and silicon. (g) Three-layer crescents of gold on the inside, silicon dioxide in the middle, and palladium on the outside. (h) Rings of lead sulfide nanocrystals obtained by sectioning an array of epoxy nanoposts drop-cast with a solution of the crystals in hexanes. Reproduced with permission from ref. [59]. Copyright 2010, American Chemical Society.

There are at least five important aspects of the structures produced by this procedure that cannot be replicated easily, if at all, with other techniques: i) the linewidths of the structures are determined by the thickness of the thin film, not the dimensions of the original topographic 
master, and thus the nanoscale dimensions do not need to be encoded by EBL or FIB to appear in the final structure; ii) the height of the structures can be tuned over a large range ( $80 \mathrm{~nm}-2 \mu \mathrm{m}$ demonstrated in Figure 13b and 13c), simply by changing the thickness of the slabs cut by the ultramicrotome; iii) the structures can comprise two or more materials in the same plane, in a single step of replication; iv) the components can be in physical contact in the lateral dimension; and v) many slabs may be obtained from a single embedded structure (we have produced as many as 60 consecutive cross sections, $100 \mathrm{~nm}$ thick, from a single embedded array of $8-\mu \mathrm{m}$, gold-coated epoxy nanoposts). ${ }^{[59]}$

\subsection{Plasmonic Properties of Two-Dimensional Arrays of Nanostructures}

In Section 4.2, we described visible LSPRs in along the short axes of gold nanowires. It is also possible to excite LSPRs around the perimeters of rings, split-rings, and L-shaped structures. ${ }^{[56]}$ Figure 14a shows a spectrum in the near-to-mid IR of two different samples: a 2D array of rings with $d=330 \mathrm{~nm}$ (dotted line), and an array of concentric rings, where the inner ring had $d=330 \mathrm{~nm}$, and the outer ring had $d=730 \mathrm{~nm}$. The single ring produced one resonance around $2.5 \mu \mathrm{m}$. The double ring produced two resonances (solid line), where the resonance due to the inner ring was higher in energy than the single ring of the same dimensions, due to the presence of the outer ring. Our experimental observations were consistent with FDTD simulations (Figure 14b). These structures behaved as short-wavelength IR frequency-selective surfaces. 

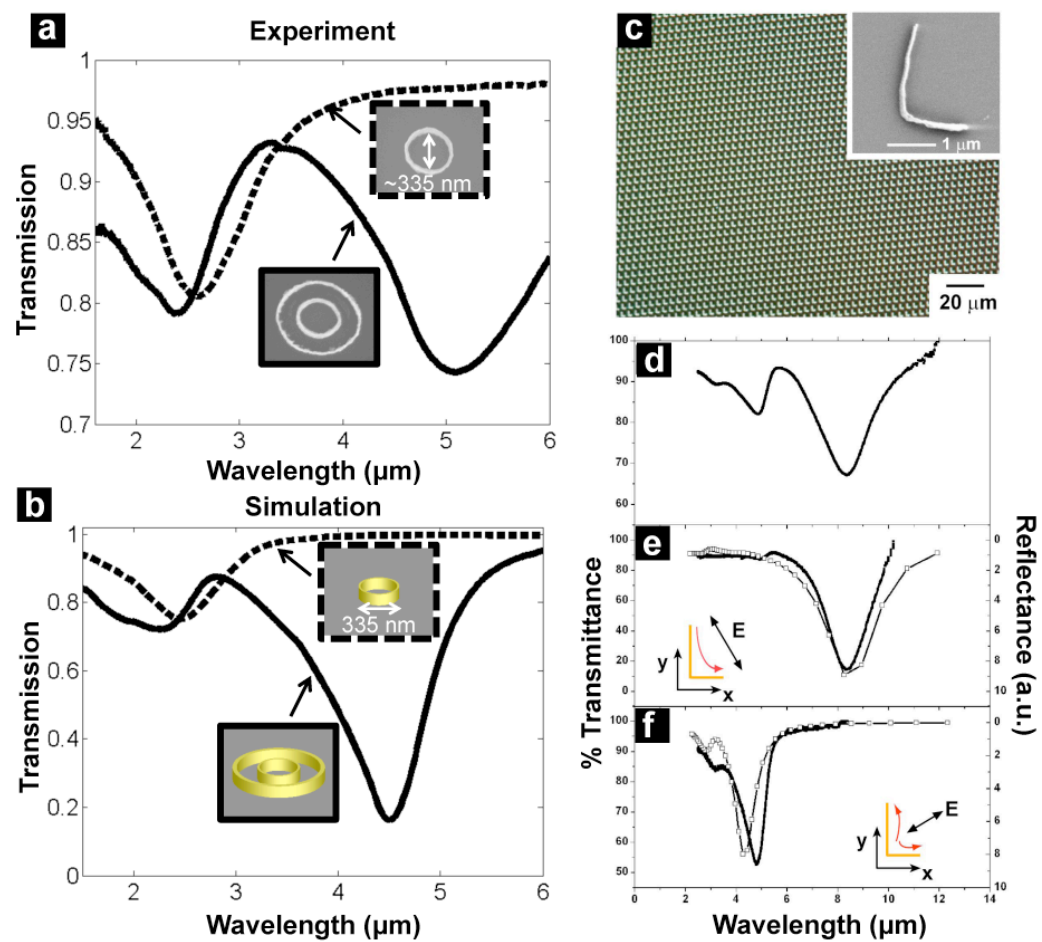

Figure 14. Infrared spectra of two-dimensional arrays of metallic nanostructures. (a) Comparison of spectra between single rings (dotted line) and double rings (solid line). (b) Finite-difference time-domain simulations of the structures measured in (a). Reproduced with permission from ref. [59]. Copyright 2010, American Chemical Society. (c) Dark-field image of an array of L-shaped structures. The inset is an SEM of an individual structure. (d) Mid-IR transmission spectrum of the array excited using unpolarized light. (e,f) Transmission spectra of the structures when the polarization is oriented (e) parallel and (f) perpendicular to the line connecting the termini of the L-shaped structures. Reproduced with permission from ref. [56]. Copyright 2007, American Chemical Society.

The arrays of rings were isotropic, and thus the resonances were not dependent on polarization. In order to test the ability to produce anisotropic structures for polarizationdependent applications, Xu et al. produced the L-shaped structures in Figure 14c by a combination of molding and line-of-sight deposition of gold. ${ }^{[56]}$ Figure 14d shows the resonance of the array in response to unpolarized light. It consists of two distinct modes. The mode at 8.4 $\mu \mathrm{m}$ was due to an oscillation in a line that connects the two termini of the L, and was excited by linearly polarized light that was parallel to that line (Figure 14e). The mode at $4.8 \mu \mathrm{m}$ was excited by light polarized perpendicular to the line that connects the two ends of the L (Figure 
14f). This polarization bisected the structure and produced two orthogonal regions that oscillated in phase. These observations were again consistent with FDTD simulations. ${ }^{[56]}$

\subsection{Integration of Plasmonic Arrays with Optical Fibers}

The thin slab of epoxy in which the structures produced by nanoskiving are embedded provides a visible handle to transfer arrays to substrates. ${ }^{[59]}$ There is, thus, a major challenge in optics to which nanoskiving seems particularly (perhaps uniquely) well-suited-modifying the cleaved facets of optical fibers with arrays of nanostructures. The ability to control the emission from fibers using filters or polarizers, or the fabrication of sensors for in situ, label-free detection of chemical or biological analytes using either SERS ${ }^{[148]}$ or LSPRs, ${ }^{[149]}$ are possible applications of modified optical fibers. ${ }^{[150]}$ Attachment of plasmonic arrays to (or formation on) the cleaved facets of fibers is not straightforward by conventional means, however. Photolithographic patterning of the facets of fibers would require deposition, exposure and development of photoresist on a small area $(d \sim 100 \mu \mathrm{m})$, with the fiber somehow positioned correctly. ${ }^{[24]}$ Examples of unconventional methods to integrate plasmonic elements with optical fibers include anisotropic chemical etching, to form arrays of sharp cones, ${ }^{[151]}$ and transferring gold structures fabricated by EBL from a surface to which gold adhered weakly. ${ }^{[124]}$

Figure 15a summarizes the procedure we used to mount arrays of metallic nanostructures on the facets of fibers. ${ }^{[152]}$ We were able to do so by submerging the floating slabs by pressing down, from above, with the tip of a fiber. This action submerged the slab, which became attached to the tip of the fiber as we withdrew it from the water-filled trough of the ultramicrotome. After allowing the water on the tip of the fiber to evaporate, exposure to an air 
plasma using a bench-top plasma cleaner (1 torr, $100 \mathrm{~W}, 10 \mathrm{~min}$ ) left behind the nanostructures only on the facet of the fiber (Figures $\mathbf{1 5 b}$ and 15c).

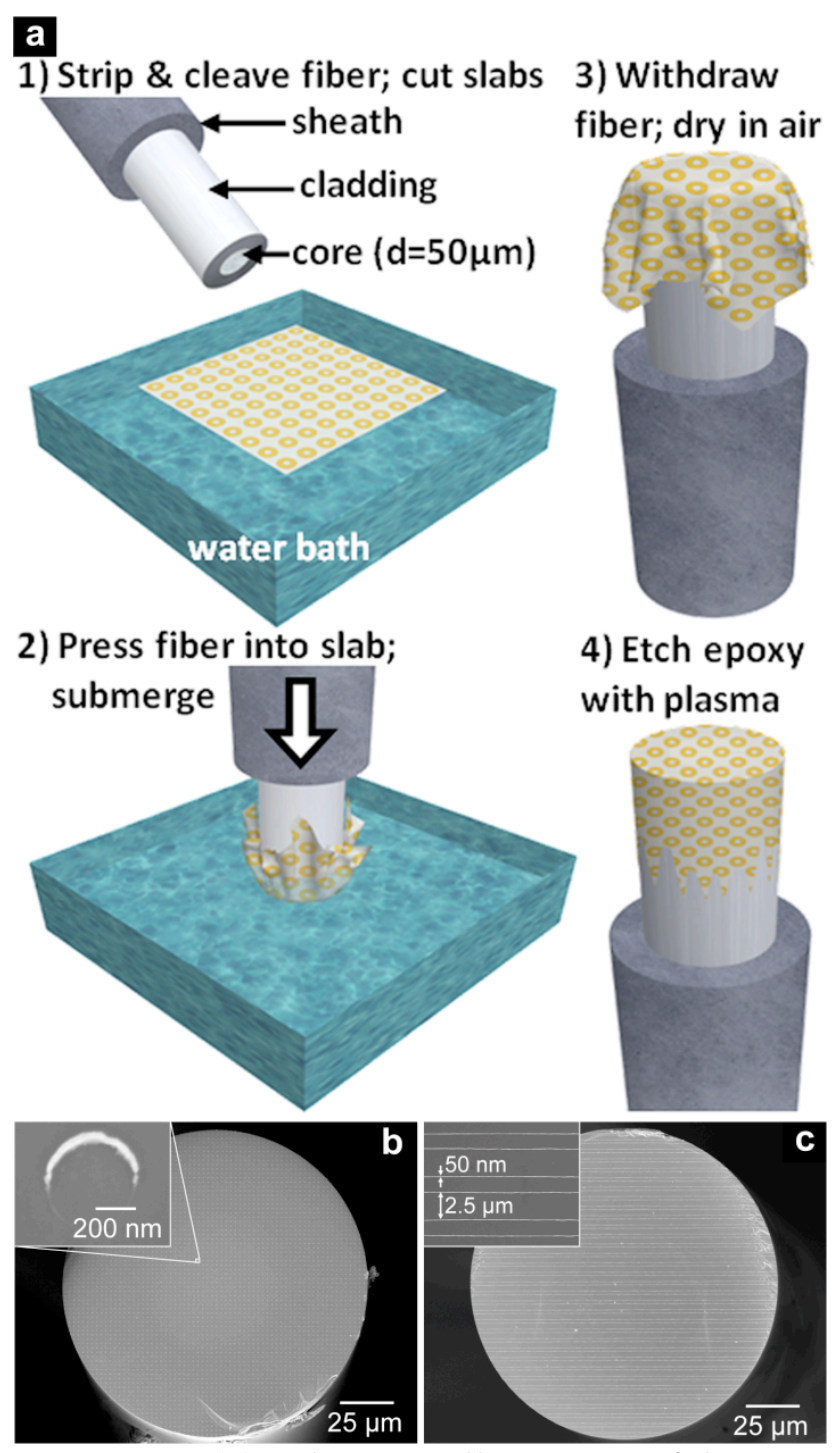

Figure 16. (a) Schematic illustration of the procedure used to transfer arrays of plasmonic nanostructures to the cleaved facet of an optical fiber. (b) A SEM image of a facet of an optical fiber bearing a square array of gold crescents. (c) A facet bearing a grating of parallel gold nanowires. Reproduced with permission from ref. [152]. Copyright 2011, American Chemical Society.

\section{Summary and Outlook}

Nanoskiving is an experimentally simple method of generating nanostructures for applications in optics and electronics, though the optical applications are presently more 
developed than are the electronic ones. The equipment it requires is much less expensive (by a factor of 10-20) and more widely available than the e-beam or FIB writers often used to make nanostructures. Nanoskiving introduces "cutting" as a step that replicates patterns and generates nanoscale features; it is analogous to "printing" and "molding" in soft lithography, and to "exposure" in photolithography. While the most complex structures produced by nanoskiving require a topographically patterned template, the nanoscale dimensions do not come from this template. Rather, they correspond to the thicknesses of thin films (evaporated, sputter-coated, spin-coated, or chemically deposited) generated on the surface of the epoxy template. In this sense, nanoskiving serves simultaneously as a technique of mastering and replication of nanoscale information. This characteristic has no analogue among the other techniques of nanofabrication.

Nanoskiving has a low barrier to entry, in terms of initial capital investments and the learning curve. The first several steps of all of the procedures discussed in this paper-e.g., soft lithographic molding and thin-film deposition — are well established in the literature and widely practiced. ${ }^{[153]} \mathrm{A}$ user can start generating high-quality nanostructures after a half-day session of training, and a few sessions of independent practice. Additional experience increases the speed with which samples can be trimmed and sectioned, but the quality of the nanostructures obtained depends most strongly on the preparation of blocks - molding, deposition, embedding, and orienting — rather than on the process of sectioning itself.

As with any technique, nanoskiving has its disadvantages. It is limited to generating noncrossing line segments (although these structures can be produced by stacking slabs). The technique works best for polymers and metals softer than platinum; extensive fragmentation of brittle materials - hard metals, crystalline oxides, and some amorphous semiconductors_limits 
its generality (though the number of materials for which nanoskiving does work is large).

Nanoskiving is subject to all of the artifacts of ultramicrotomy, the most important of which are scoring and compression. Scoring can be avoided by working in an environment uncontaminated with hard, microscopic particles of dust. Compression causes two deleterious effects. First, it distorts square arrays of nanostructures into rectangular arrays $(8.5 \%$ compression for UVO114). Second, it imposes compressive stress on embedded films that lie parallel to the direction of cutting, and these segments are prone to fragmentation. The use of oscillating knives and other methods ${ }^{[64,154]}$ will mitigate these deleterious effects as nanoskiving develops. ${ }^{[155]}$

There are several future directions of nanoskiving. In optics, the two most salient are i) the ability to fabricate structures of multiple materials and ii) the integration of arrays of metallic nanoparticles with optical fibers and other components. The fabrication of three-dimensional metamaterials is also an area of potential significance for nanoskiving. ${ }^{[130,133]}$ Stacking and laminating structures could be a route toward 3D materials with different geometries and compositions within or between layers. ${ }^{[59,74]}$ Other areas in which nanoskiving has potential are nanoelectrochemistry, ${ }^{[80]}$ patterning nanoscale magnetic particles for digital storage, ${ }^{[156]}$ membranes for size or shape-selective diffusion, ${ }^{[157]}$ devices for energy conversion and storage ${ }^{[158]}$ and patterning functional surfaces for biology. ${ }^{[159]}$

The ability to produce consecutive cross sections — quasi copies—of structures suggests that thin sectioning could be useful in manufacturing. The most significant impediment to transforming nanoskiving from a technique for research to one of manufacturing is replacing the manual steps (aligning the embedded structures with the knife edge and collecting the sections from the water-filled trough) with automated ones. A recent technological development-reel-toreel lathing ultramicrotomy — stands out as potentially useful for high-throughput and large-area 
nanoskiving. ${ }^{[160]}$ Nanoskiving might, ultimately, suggest new ways of nanomanufacturing by cutting.

\section{Acknowledgements}

This research was supported by the National Science Foundation under award PHY0646094 and by the Office of Naval Research under award N0014-10-1-0942. The authors used the shared facilities supported by the NSF under MRSEC (DMR-0213805 and DMR-0820484). This work was performed in part using the facilities of the Center for Nanoscale Systems (CNS), a member of the National Nanotechnology Infrastructure Network (NNIN), which is supported by the National Science Foundation under NSF award no. ECS-0335765. CNS is part of the Faculty of Arts and Sciences at Harvard University. D.J.L. acknowledges a Graduate Fellowship from the American Chemical Society, Division of Organic Chemistry, sponsored by Novartis.

\section{References}

[1] C. B. Murray, C. R. Kagan, M. G. Bawendi, Annu. Rev. Mater. Sci. 2000, 30, 545.

[2] P. Mulvaney, MRS Bull. 2001, 26, 1009.

[3] P. Avouris, Phys. Today 2009, 62, 34.

[4] G. M. Whitesides, D. J. Lipomi, Faraday Disc. 2009, 143, 373.

[5] R. F. Pease, S. Y. Chou, Proc. IEEE 2008, 96, 248.

[6] B. Y. S. Kim, J. T. Rutka, W. C. W. Chan N. Engl. J. Med. 2010, 363, 2434.

[7] A. V. Akimov, A. Mukherjee, C. L. Yu, D. E. Chang, A. S. Zibrov, P. R. Hemmer, H. Park, M. D. Lukin, Nature 2007, 450, 402. 
[8] E. Cubukcu, N. F. Yu, E. J. Smythe, L. Diehl, K. B. Crozier, F. Capasso, IEEE J. Sel. Top. Quantum Electron. 2008, 14, 1448.

[9] B. D. Gates, Q. B. Xu, M. Stewart, D. Ryan, C. G. Willson, Whitesides, G. M. Chem. Rev. 2005, 105, 1171.

[10] C. G. Willson, B. J. Roman, ACS Nano 2008, 2, 1323.

[11] R. K. Cavin, V. V. Zhirnov, D. J. C. Herr, A. Avila, J. Hutchby, J. Nanopart. Res. 2006, 8,841 .

[12] M. Lundstrom, Science 2003, 299, 210.

[13] M. Gower, Microlith. World 2004, 13, 16.

[14] H. Ito, J. Photopolym. Sci. Technol. 2008, 21, 475.

[15] J. Lopez-Gejo, J. T. Kunjappu, J. Zhou, B. W. Smith, P. Zimmerman, W. Conley, N. J. Turro, Chem. Mater. 2007, 19, 3641.

[16] K. Ronse, C. R. Phys. 2006, 7, 844.

[17] C. A. Mack, IEEE Spectrum 2008, 45, 40.

[18] D. Bratton, D. Yang, J. Y. Dai, C. K. Ober, Polym. Adv. Technol. 2006, 17, 94.

[19] C. N. Berglund, R. C. Leachman, IEEE Trans. Semicond. Manuf. 2010, 23, 39.

[20] C. G. Willson, J. Photopolym. Sci. Technol. 2009, 22, 147.

[21] B. J. Wiley, D. Qin, Y. N. Xia, ACS Nano 2010, 4, 3554.

[22] Y. N. Xia, E. Kim, X. M. Zhao, J. A. Rogers, M. Prentiss, G. M. Whitesides, Science 1996, $273,347$.

[23] D. H. Kim, J. A. Rogers, Adv. Mater. 2008, 20, 4887.

[24] E. J. Smythe, M. D. Dickey, G. M. Whitesides, F. Capasso, ACS Nano 2009, 3, 59. 
[25] Y. N. Xia, G. M. Whitesides, Angew. Chem., Int. Ed. 1998, 37, 551.

[26] C. Goh, K. M. Coakley, M. D. McGehee, Nano Lett. 2005, 5, 1545.

[27] Q. B. Xu, B. T. Mayers, M. Lahav, D. V. Vezenov, G. M. Whitesides, J. Am. Chem. Soc. 2005, 127, 854.

[28] Y. N. Xia, J. J. McClelland, R. Gupta, D. Qin, X. M. Zhao, L. L. Sohn, R. J. Celotta, G. M. Whitesides, Adv. Mater. 1997, 9, 147.

[29] E. Kim, Y. N. Xia, X. M. Zhao, G. M. Whitesides, Adv. Mater. 1997, 9, 651.

[30] N. L. Jeon, I. S. Choi, B. Xu, G. M. Whitesides, Adv. Mater. 1999, 11, 946.

[31] R. S. Kane, S. Takayama, E. Ostuni, D. E. Ingber, G. M. Whitesides, Biomater. 1999, 20, 2363.

[32] Y. N. Xia, G. M. Whitesides, Langmuir 1997, 13, 2059.

[33] H. A. Biebuyck, N. B. Larsen, E. Delamarche, B. Michel, IBM J. Res. Dev. 1997, $41,159$.

[34] Y. N. Xia, D. Qin, G. M. Whitesides, Adv. Mater. 1996, 8, 1015.

[35] T. B. Cao, Q. B. Xu, A. Winkleman, G. M. Whitesides, Small 2005, 1, 1191.

[36] M. Q. Xue, Y. H. Yang, T. B. Cao, Adv. Mater. 2008, 20, 596.

[37] D. J. Shir, S. Jeon, H. Liao, M. Highland, D. G. Cahill, M. F. Su, I. F. El-Kady, C. G. Christodoulou, G. R. Bogart, A. V. Hamza, J. A. Rogers, J. Phys. Chem. B 2007, 111, 12945.

[38] J. Maria, S. Jeon, J. A. Rogers, J. Photochem. Photobiol., A 2004, 166, 149.

[39] J. A. Rogers, K. E. Paul, R. J. Jackman, G. M. Whitesides, Appl. Phys. Lett. 1997, $70,2658$.

[40] S. Y. Chou, P. R. Krauss, P. J. Renstrom, Appl. Phys. Lett. 1995, 67, 3114.

[41] S. Y. Chou, P. R. Krauss, P. J. Renstrom, Science 1996, $272,85$. 
[42] T. C. Bailey, S. C. Johnson, S. V. Sreenivasan, J. G. Ekerdt, C. G. Willson, D. J. Resnick, J. Photopolym. Sci. Tech. 2002, 15, 481.

[43] R. Garcia, R. V. Martinez, J. Martinez, Chem. Soc. Rev. 2006, 35, 29.

[44] W. Shim, A. B. Braunschweig, X. Liao, J. N. Chai, J. K. Lim, G. F. Zheng, C. A. Mirkin Nature 2011, 469, 516.

[45] K. Salaita, Y. H. Wang, J. Fragala, R. A. Vega, C. Liu, C. A. Mirkin, Angew. Chem., Int. Ed. 2006, 45, 7220.

[46] L. G. Rosa, J. Liang, J. Phys. Condens. Matter 2009, 21, 483001.

[47] J. L. Gong, D. J. Lipomi, J. D. Deng, Z. H. Nie, X. Chen, N. X. Randall, R. Nair, G. M. Whitesides, Nano Lett. 2010, 10, 2702.

[48] T. F. Malis, D. Steele, Mater. Res. Soc. Symp. Proc. 1990, 199, 3.

[49] W. Villiger, A. Bremer, J. Struct. Biol. 1990, 104, 178.

[50] Q. B. Xu, R. M. Rioux, M. D. Dickey, G. M. Whitesides, Acc. Chem. Res. 2008, $41,1566$.

[51] Hill, J. The Construction of Timber; Imperial Academy: London, 1770.

[52] D. C. Pease, K. R. Porter, J. Cell Biol. 1981, 91, S287.

[53] B. M. Bracegirdle, A History of Microtechnique; Cornell University Press: Ithaca, N. Y., 1978.

[54] J. N. Goldstein, D. Newbury, D. Joy, C. Lyman, P. Echlin, E. Lifshin, L. Sawyer, J. Michael, Scanning Electron Microscopy and X-Ray Analysis; 3 ed.; Springer, 2003.

[55] J. D. Acetarin, E. Carlemalm, E. Kellenberger, W. Villiger, J. Electron Microsc. Tech. 1987, 6, 63. 
[56] Q. B. Xu, J. M. Bao, R. M. Rioux, R. Perez-Castillejos, F. Capasso, G. M.

Whitesides, Nano Lett. 2007, 7, 2800.

[57] D. J. Lipomi, R. V. Martinez, R. M. Rioux, L. Cademartiri, W. F. Reus, G. M. Whitesides, ACS Appl. Mater. Interfaces 2010, 2, 2503.

[58] A. Antonovsky, Microsc. Res. Tech. 1995, 31, 300.

[59] D. J. Lipomi, M. A. Kats, P. Kim, S. H. Kang, J. Aizenberg, F. Capasso, G. M. Whitesides, ACS Nano 2010, 4, 4017.

[60] Q. Xu, R. M. Rioux, G. M. Whitesides, ACS Nano 2007, 1, 215.

[61] B. J. Wiley, D. J. Lipomi, J. M. Bao, F. Capasso, Whitesides, Nano Lett. 2008, 8, 3023.

[62] D. J. Lipomi, R. C. Chiechi, W. F. Reus, G. M. Whitesides, Adv. Funct. Mater. 2008, $18,3469$.

[63] T. R. Matzelle, H. Gnaegi, A. Ricker, R. Reichelt, J. Microsc. Oxford 2003, 209, 113.

[64] J. C. Jesior, J. Ultrastruct. Mol. Struct. Res. 1986, 95, 210.

[65] Q. B. Xu, J. M. Bao, F. Capasso, G. M. Whitesides, Angew. Chem., Int. Ed. 2006, 45,3631 .

[66] Y. Xia, Y. J. Xiong, B. Lim, S. E. Skrabalak, Angew. Chem., Int. Ed. 2009, 48, 60.

[67] C. Burda, X. B. Chen, R. Narayanan, M. A. El-Sayed, Chem. Rev. 2005, 105, 1025.

[68] J. Robertson, Rep. Prog. Phys. 2006, 69, 327.

[69] A. K. Geim, K. S. Novoselov, Nat. Mater. 2007, 6, 183. 
[70] Y. N. Xia, P. D. Yang, Y. G. Sun, Y. Y. Wu, B. Mayers, B. Gates, Y. D. Yin, F. Kim, Y. Q. Yan, Adv. Mater. 2003, 15, 353.

[71] A. L. Falk, F. H. L. Koppens, C. L. Yu, K. Kang, N. D. Snapp, A. V. Akimov, M. H. Jo, M. D. Lukin, H. Park, Nat. Phys. 2009, 5, 475.

[72] Q. B. Xu, B. D. Gates, G. M. Whitesides, J. Am. Chem. Soc. 2004, 126, 1332.

[73] M. D. Dickey, D. J. Lipomi, P. J. Bracher, G. M. Whitesides, Nano Lett. 2008, 8, 4568.

[74] D. J. Lipomi, F. Ilievski, B. J. Wiley, P. B. Deotare, M. Lonçar, G. M. Whitesides, ACS Nano 2009, 3, 3315.

[75] D. J. Lipomi, R. C. Chiechi, M. D. Dickey, G. M. Whitesides, Nano Lett. 2008, 8, 2100.

[76] K. Ramanathan, M. A. Bangar, M. H. Yun, W. F. Chen, A. Mulchandani, N. V. Myung, Nano Lett. 2004, 4, 1237.

[77] M. H. Yun, N. V. Myung, R. P. Vasquez, C. S. Lee, E. Menke, R. M. Penner, Nano Lett. 2004, 4, 419.

[78] A. Bezryadin, C. Dekker, G. Schmid, Appl. Phys. Lett. 1997, 71, 1273.

[79] R. Holzel, N. Calander, Z. Chiragwandi, M. Willander, F. F. Bier, Phys. Rev. Lett. 2005, 95 .

[80] R. W. Murray, Chem. Rev. 2008, 108, 2688.

[81] N. J. Tao, Nat. Nanotechnol. 2006, 1, 173.

[82] E. A. Weiss, J. K. Kriebel, M. A. Rampi, G. M. Whitesides, Philos. Trans. R. Soc. London, Ser. A 2007, 365, 1509. 
[83] J. B. Lassiter, J. Aizpurua, L. I. Hernandez, D. W. Brandl, I. Romero, S. Lal, J. H. Hafner, P. Nordlander, N. J. Halas, Nano Lett. 2008, 8, 1212.

[84] H. Q. Liu, J. Kameoka, D. A. Czaplewski, H. G. Craighead, Nano Lett. 2004, 4, 671.

[85] D. T. McQuade, A. E. Pullen, T. M. Swager, Chem. Rev. 2000, 100, 2537.

[86] E. C. Walter, F. Favier, R. M. Penner, Anal. Chem. 2002, 74, 1546.

[87] E. C. Walter, B. J. Murray, F. Favier, G. Kaltenpoth, M. Grunze, R. M. Penner, J. Phys. Chem. B 2002, 106, 11407.

[88] E. A. Weiss, G. K. Kaufman, J. K. Kriebel, Z. Li, R. Schalek, G. M. Whitesides, Langmuir 2007, 23, 9686.

[89] M. E. Roberts, A. N. Sokolov, Z. N. Bao, J. Mater. Chem. 2009, 19, 3351.

[90] K. Ramanathan, M. A. Bangar, M. Yun, W. Chen, N. V. Myung, A. Mulchandani, J. Am. Chem. Soc. 2005, 127, 496.

[91] J. L. Duvail, P. Retho, V. Fernandez, G. Louarn, P. Molinie, O. Chauvet, J. Phys. Chem. B 2004, 108, 18552.

[92] H. Q. Liu, C. H. Reccius, H. G. Craighead, Appl. Phys. Lett. 2005, 87.

[93] E. Smela, Adv. Mater. 2003, 15, 481.

[94] S. Samitsu, T. Shimomura, K. Ito, M. Fujimori, S. Heike, T. Hashizume, Appl. Phys. Lett. 2005, 86.

[95] B. Dong, N. Lu, M. Zelsmann, N. Kehagias, H. Fuchs, C. M. S. Torres, L. F. Chi, Adv. Funct. Mater. 2006, 16, 1937.

[96] A. Greiner, J. H. Wendorff, Angew. Chem., Int. Ed. 2007, 46, 5670. 
[97] J. Kameoka, D. Czaplewski, H. Q. Liu, H. G. Craighead, J. Mater. Chem. 2004, $14,1503$.

[98] H. Q. Liu, J. Kameoka, D. A. Czaplewski, H. G. Craighead, Nano Lett. 2004, 4, 671.

[99] G. Li, V. Shrotriya, J. S. Huang, Y. Yao, T. Moriarty, K. Emery, Y. Yang, Nat. Mater. 2005, 4, 864.

[100] J. T. McCann, J. I. L. Chen, D. Li, Z. G. Ye, Y. N. Xia, Chem. Phys. Lett. 2006, $424,162$.

[101] C. A. Stover, D. L. Koch, C. Cohen, J. Fluid Mech. 1992, 238, 277.

[102] Z. H. Zhong, D. L. Wang, Y. Cui, M. W. Bockrath, C. M. Lieber, Science 2003, $302,1377$.

[103] Y. Cui, C. M. Lieber, Science 2001, 291, 851.

[104] Y. Huang, X. F. Duan, Q. Q. Wei, C. M. Lieber, Science 2001, 291, 630.

[105] B. Messer, J. H. Song, P. D. Yang, J. Am. Chem. Soc. 2000, 122, 10232.

[106] G. H. Yu, A. Y. Cao, C. M. Lieber, Nat. Nanotechnol. 2007, 2, 372.

[107] Z. Y. Fan, J. C. Ho, Z. A. Jacobson, R. Yerushalmi, R. L. Alley, H. Razavi, A. Javey, Nano Lett. 2008, 8, 20.

[108] S. Jin, D. M. Whang, M. C. McAlpine, R. S. Friedman, Y. Wu, C. M. Lieber, Nano Lett. 2004, 4, 915.

[109] P. J. Pauzauskie, A. Radenovic, E. Trepagnier, H. Shroff, P. D. Yang, J. Liphardt, Nat. Mater. 2006, 5, 97.

[110] A. Jamshidi, P. J. Pauzauskie, P. J. Schuck, A. T. Ohta, P. Y. Chiou, J. Chou, P. D. Yang, M. C. Wu, Nat. Photonics 2008, 2, 85. 
[111] H. W. C. Postma, A. Sellmeijer, C. Dekker, Adv. Mater. 2000, 12, 1299.

[112] D. J. Sirbuly, M. Law, P. Pauzauskie, H. Q. Yan, A. V. Maslov, K. Knutsen, C. Z. Ning, R. J. Saykally, P. D. Yang, Proc. Natl. Acad. Sci. USA 2005, 102, 7800.

[113] P. A. Smith, C. D. Nordquist, T. N. Jackson, T. S. Mayer, B. R. Martin, J. Mbindyo, T. E. Mallouk, Appl. Phys. Lett. 2000, 77, 1399.

[114] M. Q. Xue, Y. Zhang, Y. L. Yang, T. B. Cao, Adv. Mater. 2008, 20, 2145.

[115] R. D. McCullough, Adv. Mater. 1998, 10, 93.

[116] B. C. Thompson, J. M. J. Fréchet, Angew. Chem., Int. Ed. 2008, 47, 58.

[117] K. M. Coakley, M. D. McGehee, Chem. Mater. 2004, 16, 4533.

[118] S. A. Maier, H. A. Atwater, J. Appl. Phys. 2005, 98.

[119] M. E. Stewart, C. R. Anderton, L. B. Thompson, J. Maria, S. K. Gray, J. A.

Rogers, R. G. Nuzzo, Chem. Rev. 2008, 108, 494.

[120] J. C. Love, K. E. Paul, G. M. Whitesides, Adv. Mater. 2001, 13, 604.

[121] D. M. Wu, N. Fang, C. Sun, X. Zhang, W. J. Padilla, D. N. Basov, D. R. Smith, S. Schultz, Appl. Phys. Lett. 2003, 83, 201.

[122] A. K. Sharma, R. Jha, B. D. Gupta, IEEE Sens. J. 2007, 7, 1118.

[123] K. Kneipp, H. Kneipp, I. Itzkan, R. R. Dasari, M. S. Feld, J. Phys. Condens.

Matter 2002, 14, R597.

[124] E. J. Smythe, M. D. Dickey, J. M. Bao, G. M. Whitesides, F. Capasso, Nano Lett. 2009, 9, 1132.

[125] X. F. Liu, C. H. Sun, N. C. Linn, B. Jiang, P. Jiang, J. Phys. Chem. C 2009, 113, 14804.

[126] E. Fort, S. Gresillon, J. Phys. D, Appl. Phys. 2008, 41. 
[127] P. Peumans, V. Bulovic, S. R. Forrest, Appl. Phys. Lett. 2000, 76, 2650.

[128] T. A. Klar, A. V. Kildishev, V. P. Drachev, V. M. Shalaev, IEEE J. Sel. Top. Quantum Electron. 2006, 12, 1106.

[129] J. B. Pendry, A. J. Holden, D. J. Robbins, W. J. Stewart, IEEE Trans. Microwave Theory Tech. 1999, 47, 2075.

[130] J. Valentine, S. Zhang, T. Zentgraf, E. Ulin-Avila, D. A. Genov, G. Bartal, X. Zhang, Nature 2008, 455, 376.

[131] J. B. Pendry, Phys. Rev. Lett. 2000, 85, 3966.

[132] J. B. Pendry, D. Schurig, D. R. Smith, Science 2006, 312, 1780.

[133] N. Liu, H. C. Guo, L. W. Fu, S. Kaiser, H. Schweizer, H. Giessen, Nat. Mater. 2008, 7, 31 .

[134] J. K. Gansel, M. Thiel, M. S. Rill, M. Decker, K. Bade, V. Saile, G. von Freymann, S. Linden, M. Wegener, Science 2009, 325, 1513.

[135] A. Ahmadi, S. Ghadarghadr, H. Mosallaei, Opt. Express 2010, 18, 123.

[136] C. L. Haynes, R. P. Van Duyne, J. Phys. Chem. B 2001, 105, 5599.

[137] M. C. Gwinner, E. Koroknay, L. W. Fu, P. Patoka, W. Kandulski, M. Giersig, H. Giessen, Small 2009, 5, 400.

[138] K. E. Paul, C. Zhu, J. C. Love, G. M. Whitesides, Appl. Opt. 2001, 40, 4557.

[139] M. E. Stewart, N. H. Mack, V. Malyarchuk, J. Soares, T. W. Lee, S. K. Gray, R. G. Nuzzo, J. A. Rogers, Proc. Natl. Acad. Sci. USA 2006, 103, 17143.

[140] J. Henzie, J. E. Barton, C. L. Stender, T. W. Odom, Acc. Chem. Res. 2006, 39, 249.

[141] J. Lee, W. Hasan, C. L. Stender, T. W. Odom, Acc. Chem. Res. 2008, 41, 1762. 
[142] A. L. Pyayt, B. J.; Wiley, Y. N. Xia, A. Chen, L. Dalton, Nat. Nanotechnol. 2008, 3,660 .

[143] P. Nagpal, N. C. Lindquist, S. H. Oh, D. J. Norris, Science 2009, 325, 594.

[144] H. Ditlbacher, A. Hohenau, D. Wagner, U. Kreibig, M. Rogers, F. Hofer, F. R. Aussenegg, J. R. Krenn, Phys. Rev. Lett. 2005, 95.

[145] M. Allione, V. V. Temnov, Y. Fedutik, U. Woggon, M. V. Artemyev, Nano Letters 2008, 8, 31 .

[146] A. Graff, D. Wagner, H. Ditlbacher, U. Kreibig, Eur. Phys. J. D 2005, 34, 263.

[147] C. X. Kan, X. G. Zhu, G. H. Wang, J. Phys. Chem. B 2006, 110, 4651.

[148] A. Lucotti, G. Zerbi, Sens. Actuators, B 2007, 121, 356.

[149] A. K. Sharma, R. Jha, B. D. Gupta, IEEE Sens. J. 2007, 7, 1118.

[150] A. Leung, P. M. Shankar, R. Mutharasan, Sens. Actuators, B 2007, 125, 688.

[151] V. Guieu, D. Talaga, L. Servant, N. Sojic, F. Lagugne-Labarthet, J. Phys. Chem. C 2009, 113, 874 .

[152] D. J. Lipomi, R. V. Martinez, M. A. Kats, S. H. Kang, P. Kim, J. Aizenberg, F. Capasso, G. M. Whitesides, Nano Lett. 2011, 11, 632-636.

[153] D. Qin, Y. N. Xia, G. M. Whitesides, Nat. Protoc. 2010, 5, 491.

[154] J. C. Jesior, J. Ultrastruct. Res. 1985, 90, 135.

[155] D. Studer, H. Gnaegi, J. Microsc. Oxford 2000, 197, 94.

[156] J. Shi, S. Gider, K. Babcock, D. D. Awschalom, Science 1996, 271, 937.

[157] N. B. McKeown, P. M. Budd, Chem. Soc. Rev. 2006, 35, 675.

[158] K. Shah, W. C. Shin, R. S. Besser, Sens. Actuators, B 2004, 97, 157. 
[159] D. B. Weibel, W. R. DiLuzio, G. M. Whitesides, Nat. Rev. Microbiol. 2007, 5, 209.

[160] N. Kasthuri, K. Hayworth, J. C. Tapia, R. Schalek, S. Nundy, J. W. Lichtman, Soc. Neurosci. Abstr. 2009. 